\title{
On the uniform convergence of double sine series
}

\author{
K. Duzinkiewicz ${ }^{1}$ and B. Szal ${ }^{2}$ \\ University of Zielona Góra \\ Faculty of Mathematics, Computer Science and Econometrics \\ 65-516 Zielona Góra, ul. Szafrana 4a, Poland \\ ${ }^{1} \mathrm{~K}$.Duzinkiewicz@wmie.uz.zgora.pl \\ ${ }^{2}$ B.Szal@wmie.uz.zgora.pl
}

\begin{abstract}
The fundamental theorem in the theory of the uniform convergence of sine series is due to Chaundy and Jolliffe from 1916 (see [1). Several authors gave conditions for this problem supposing that coefficients are monotone, non-negative or more recently, general monotone (see [8, 6] and [2], for example). There are also results for the regular convergence of double sine series to by uniform in case the coefficients are monotone or general monotone double sequences. In this article we give new sufficient conditions for the uniformity of the regular convergence of double sine series, which are necessary as well in case the coefficients are non-negative. We shall generalize those results defining a new class of double sequences for the coefficients.
\end{abstract}

Mathematics subject classification number: 42A20, 42A32, $42 \mathrm{~B} 99$.

Key words: double sine series, uniform convergence, generalized monotonicity.

\section{Known results: uniform convergence of single sine series}

Let $\left\{c_{k}\right\}_{k=1}^{\infty}$ be a non-negative real sequence and consider the series

$$
\sum_{k=1}^{\infty} c_{k} \sin k x .
$$

In 1916 Chaundy and Jolliffe 1] proved the following classical result.

Theorem 1. If $\left\{c_{k}\right\}_{k=1}^{\infty} \subset \mathbb{R}_{+}$is decreasing to zero, then (1.1) converges uniformly in $x$ if and only if

$$
k c_{k} \rightarrow 0 \quad \text { as } \quad k \rightarrow \infty \text {. }
$$

Several classes of sequences have been introduced to generalize Theorem 1 (see [10, 9], 2], 6]). These classes are larger than the class of monotone sequences and contain sequences of complex numbers as well. The definitions of the latest classes are the following:

$$
\begin{aligned}
& M V B V S=\left\{\left\{c_{k}\right\}_{k=1}^{\infty} \subset \mathbb{C}, \exists \mathcal{C}>0, \lambda \geq 2: \sum_{k=n}^{2 n}\left|\Delta_{1} c_{k}\right| \leq \frac{\mathcal{C}}{n} \sum_{k=\lceil n / \lambda\rceil}^{\lceil\lambda\rceil}\left|c_{k}\right|, n \geq \lambda\right\}, \\
& S B V S=\left\{\left\{c_{k}\right\}_{k=1}^{\infty} \subset \mathbb{C}, \exists \mathcal{C}>0, \lambda \geq 2: \sum_{k=n}^{2 n-1}\left|\Delta_{1} c_{k}\right| \leq \frac{\mathcal{C}}{n}\left(\sup _{m \geq\lceil n / \lambda\rceil} \sum_{k=m}^{2 m}\left|c_{k}\right|\right), n \geq \lambda\right\}, \\
& S B V S_{2}=\left\{\left\{c_{k}\right\}_{k=1}^{\infty} \subset \mathbb{C}, \exists \mathcal{C}>0, b(k) \nearrow \infty: \sum_{k=n}^{2 n-1}\left|\Delta_{1} c_{k}\right| \leq \frac{\mathcal{C}}{n}\left(\sup _{m \geq b(n)} \sum_{k=m}^{2 m}\left|c_{k}\right|\right), n \geq 1\right\}, \\
& G M(\beta, r)=\left\{\left\{c_{k}\right\}_{k=1}^{\infty} \subset \mathbb{C}, \exists \mathcal{C}>0: \sum_{k=n}^{2 n-1}\left|\Delta_{r} a_{k}\right| \leq \mathcal{C} \beta_{n}, n \geq 1\right\},
\end{aligned}
$$


where $\Delta_{r} c_{k}=c_{k}-c_{k+r}$ for $r \in \mathbb{N}$, and the constants $\mathcal{C}$ and $\lambda$ depend only on $\left\{c_{k}\right\}$, a sequence $\{b(k)\}_{k=1}^{\infty} \subset \mathbb{R}_{+}$ is increasing and $\beta:=\left(\beta_{k}\right)$ is a non-negative sequence. It was proved in [2] that $M V B V S \varsubsetneqq S B V S \varsubsetneqq S B V S_{2}$ and a series (1.1) with coefficients of complex numbers from the classes $M V B V S, S B V S, S B V S_{2}$, is uniformly convergent if (1.2) is satisfied. In [6] Szal showed that the class $G M\left(\beta^{*}, r\right)$, with $\beta^{*}=\beta_{n}=\frac{1}{n} \sum_{k=\lceil n / \lambda\rceil}^{\lceil\lambda n\rceil}\left|c_{k}\right|(\lambda>1)$ and $r=2$ is larger than $M V B V S$ and a series (1.1) with coefficients from $G M\left(\beta^{*}, 2\right)$ is uniformly convergent if (1.2) holds. However, the necessity of condition (1.2) for the uniform convergence of (1.1) is proved for sine series with coefficients of non-negative numbers from one of the above classes.

\section{Known results: uniform convergence of double sine series}

We start this section by giving some definitions and notations.

A double series

$$
\sum_{j=1}^{\infty} \sum_{k=1}^{\infty} c_{j k}
$$

of complex numbers converge regularly if the sum

$$
\sum_{j=1}^{m} \sum_{k=1}^{n} c_{j k}
$$

converge to a finite number as $m$ and $n$ tend to infinity independently of each other, moreover, both the column series and row series

$$
\sum_{j=1}^{\infty} z_{j n}, \quad n=1,2, \ldots, \quad \text { and } \quad \sum_{k=1}^{\infty} z_{m k} \quad m=1,2, \ldots
$$

are convergent. Or equivalently, if for any $\epsilon>0$ there exists a positive number $m_{0}=m_{0}(\epsilon)$ such that

$$
\left|\sum_{j=m}^{M} \sum_{k=n}^{N} z_{j k}\right|<\epsilon
$$

holds for any $m, n, M, N$ for which $m+n>m_{0}, 1 \leq m \leq M$ and $1 \leq n \leq N$.

A monotonically decreasing double sequence $\left\{c_{j k}\right\}_{j, k=1}^{\infty}$ is a sequence of real numbers such that

$$
\Delta_{10} c_{j k} \geq 0, \quad \Delta_{01} c_{j k} \geq 0, \quad \Delta_{11} c_{j k} \geq 0, \quad j, k=1,2, \ldots,
$$

where

$$
\begin{aligned}
& \Delta_{10} c_{j k}:=c_{j k}-c_{j+1, k}, \quad \Delta_{01} c_{j k}:=c_{j k}-c_{j, k+1}, \\
& \Delta_{11} c_{j k}:=\Delta_{10}\left(\Delta_{01} c_{j k}\right)=\Delta_{01}\left(\Delta_{10} c_{j k}\right)=c_{j k}-c_{j+1, k}-c_{j, k+1}+c_{j+1, k+1} .
\end{aligned}
$$

Let $\left\{c_{j k}\right\}_{j, k=1}^{\infty}$ be a double sequence of complex numbers. Consider the double sine series

$$
\sum_{j=1}^{\infty} \sum_{k=1}^{\infty} c_{j k} \sin j x \sin k y
$$

The two-dimensional extension of Theorem 1 is due to Žak and Šneider.

Theorem 2. ([11]) If $\left\{c_{j k}\right\}_{j, k=1}^{\infty} \subset \mathbb{R}_{+}$is a monotonicity decreasing double sequence, then (2.1) is uniformly regularly convergent in $(x, y)$ if and only if

$$
j k c_{j k} \rightarrow 0 \quad \text { as } \quad j+k \rightarrow \infty .
$$

Theorem 2 was generalized by Kórus and Móricz [3] and by Kórus [4] (and also by Leindler [5]). They have defined new classes of double sequences to obtain those generalizations. We present below those definitions and their results. 
Definition 1. ([3]) $A$ double sequence $\left\{c_{j k}\right\}_{j, k=1}^{\infty} \subset \mathbb{C}$ belongs to the class $M V B V D S$, if there exist positive constants $\mathcal{C}$ and $\lambda \geq 2$, depending only on $\left\{c_{j k}\right\}$, such that:

$$
\begin{gathered}
\sum_{j=m}^{2 m-1}\left|\Delta_{10} c_{j n}\right| \leq \frac{\mathcal{C}}{m} \sum_{\lceil j=m / \lambda\rceil}^{\lceil\lambda m\rceil}\left|c_{j n}\right|, m \geq \lambda, n \geq 1, \\
\sum_{k=n}^{2 n-1}\left|\Delta_{01} c_{k m}\right| \leq \frac{\mathcal{C}}{n} \sum_{\lceil k=n / \lambda\rceil}^{\lceil\lambda n\rceil}\left|c_{k m}\right|, n \geq \lambda, m \geq 1, \\
\sum_{j=m}^{2 m-1} \sum_{k=n}^{2 n-1}\left|\Delta_{11} c_{j k}\right| \leq \frac{\mathcal{C}}{m n} \sum_{\lceil j=m / \lambda\rceil}^{\lceil\lambda m\rceil} \sum_{\lceil k=n / \lambda\rceil}^{\lceil\lambda n\rceil}\left|c_{j k}\right|, m, n \geq \lambda .
\end{gathered}
$$

Theorem 3. ([3])

(i) If $\left\{c_{j k}\right\}_{j, k=1}^{\infty} \subset \mathbb{C}$ belongs to the class $M V B V D S$ and (2.2) holds, then 2.1) converges regularly, uniformly in $(x, y)$.

(ii) Conversely, if $\left\{c_{j k}\right\}_{j, k=1}^{\infty} \subset \mathbb{R}_{+}$belongs to the class $M V B V D S$ and (2.1) is uniformly regularly convergent in $(x, y)$, then (2.2) is satisfied.

Definition 2. ([4]) A double sequence $\left\{c_{j k}\right\}_{j, k=1}^{\infty} \subset \mathbb{C}$ belongs to the class $S B V D S_{1}$, if there exist positive constants $\mathcal{C}$ and integer $\lambda \geq 2$ and sequences $\left\{b_{1}(l)\right\}_{l=1}^{\infty},\left\{b_{2}(l)\right\}_{l=1}^{\infty},\left\{b_{3}(l)\right\}_{l=1}^{\infty}$, each one tends (not necessarily monotonically) to infinity, all of them depending only on $\left\{c_{j k}\right\}$, such that:

$$
\begin{gathered}
\sum_{j=m}^{2 m-1}\left|\Delta_{10} c_{j n}\right| \leq \frac{\mathcal{C}}{m}\left(\max _{b_{1}(m) \leq M \leq \lambda b_{1}(m)} \sum_{j=M}^{2 M}\left|c_{j n}\right|\right), m \geq \lambda, n \geq 1, \\
\sum_{k=n}^{2 n-1}\left|\Delta_{01} c_{k m}\right| \leq \frac{\mathcal{C}}{n}\left(\max _{b_{2}(n) \leq M \leq \lambda b_{2}(n)} \sum_{k=N}^{2 N}\left|c_{k m}\right|\right), n \geq \lambda, m \geq 1, \\
\sum_{j=m}^{2 m-1} \sum_{k=n}^{2 n-1}\left|\Delta_{11} c_{j k}\right| \leq \frac{\mathcal{C}}{m n}\left(\sup _{M+N \geq b_{3}(m+n)} \sum_{j=M}^{2 M} \sum_{k=N}^{2 N}\left|c_{j k}\right|\right), m, n \geq \lambda .
\end{gathered}
$$

Theorem 4. ([4]) If $\left\{c_{j k}\right\}_{j, k=1}^{\infty} \subset \mathbb{R}_{+}$belongs to the class $S B V D S_{1}$ and (2.1) is uniform regularly convergent in $(x, y)$, then (2.2) is satisfied.

Definition 3. ([4]) A double sequence $\left\{c_{j k}\right\}_{j, k=1}^{\infty} \subset \mathbb{C}$ belongs to the class $S B V D S_{2}$, if there exist positive constants $\mathcal{C}$ and integer $\lambda \geq 1$ and sequence $\{b(l)\}_{l=1}^{\infty}$ tending monotonically to infinity, depending only on $\left\{c_{j k}\right\}$, for which:

$$
\begin{aligned}
\sum_{j=m}^{2 m-1}\left|\Delta_{10} c_{j n}\right| & \leq \frac{\mathcal{C}}{m}\left(\sup _{M \geq b(m)} \sum_{j=M}^{2 M}\left|c_{j n}\right|\right), m \geq \lambda, n \geq 1, \\
\sum_{k=n}^{2 n-1}\left|\Delta_{01} c_{k m}\right| & \leq \frac{\mathcal{C}}{n}\left(\sup _{N \geq b(n)} \sum_{k=N}^{2 N}\left|c_{k m}\right|\right), n \geq \lambda, m \geq 1, \\
\sum_{j=m}^{2 m-1} \sum_{k=n}^{2 n-1}\left|\Delta_{11} c_{j k}\right| & \leq \frac{\mathcal{C}}{m n}\left(\sup _{M+N \geq b(m+n)} \sum_{j=M}^{2 M} \sum_{k=N}^{2 N}\left|c_{j k}\right|\right), m, n \geq \lambda .
\end{aligned}
$$

Theorem 5. ([4]) If $\left\{c_{j k}\right\}_{j, k=1}^{\infty} \subset \mathbb{C}$ belongs to the class $S B V D S_{2}$ and (2.2) holds, then the regularly convergent of (2.1) is uniform in $(x, y)$.

Theorem 6. (4]) $M V B V D S \varsubsetneqq S B V D S_{1} \varsubsetneqq S B V D S_{2}$.

Now, we shall define a new class of double sequences in the following way: Let, for $r \in \mathbb{N}$,

$$
\Delta_{r 0} c_{j k}:=c_{j k}-c_{j+r, k}, \Delta_{0 r} c_{j k}:=c_{j k}-c_{j, k+r}
$$

and

$$
\Delta_{r r} c_{j k}:=\Delta_{r 0}\left(\Delta_{0 r} c_{j k}\right)
$$


Definition 4. A double sequence $\left\{c_{j k}\right\}_{j, k=1}^{\infty} \subset \mathbb{C}$ belongs to the class DGM( $\left.\alpha, \beta, \gamma, r\right)$ (called Double General Monotone), if there exist positive constants $\mathcal{C}$ and integer $\lambda$ depending only on $\left\{c_{j k}\right\}$, for which:

$$
\begin{aligned}
& \sum_{j=m}^{2 m-1}\left|\Delta_{r 0} c_{j n}\right| \leq \mathcal{C} \alpha_{m n}, m \geq \lambda, n \geq 1, \\
& \sum_{k=n}^{2 n-1}\left|\Delta_{0 r} c_{k m}\right| \leq \mathcal{C} \beta_{m n}, \quad n \geq \lambda, m \geq 1, \\
& \sum_{j=m}^{2 m-1} \sum_{k=n}^{2 n-1}\left|\Delta_{r r} c_{j k}\right| \leq \mathcal{C} \gamma_{m n}, m, n \geq \lambda .
\end{aligned}
$$

hold, where $\alpha:=\left\{\alpha_{m n}\right\}_{m, n=1}^{\infty}, \beta:=\left\{\beta_{m n}\right\}_{m, n=1}^{\infty}, \gamma:=\left\{\gamma_{m n}\right\}_{m, n=1}^{\infty}$ are non-negative double sequences and $r \in \mathbb{N}$.

Using our definition for $r=1$, we have:

1) $M V B V D S \equiv D G M\left({ }_{1} \alpha,{ }_{1} \beta,{ }_{1} \gamma, 1\right)$, where $\left\{{ }_{1} \alpha\right\},\left\{{ }_{1} \beta\right\}$ and $\left\{{ }_{1} \gamma\right\}$ are the sequences defined by the formulas on the right sides of the inequalities (2.3), (2.4) and (2.5), respectively;

2) $S B V D S_{1} \equiv D G M\left({ }_{2} \alpha,{ }_{2} \beta,{ }_{2} \gamma, 1\right)$, where $\left\{{ }_{2} \alpha\right\},\left\{{ }_{2} \beta\right\}$ and $\left\{{ }_{2} \gamma\right\}$ are the sequences defined by the formulas on the right sides of the inequalities (2.6), (2.7) and (2.8), respectively;

3) $S B V D S_{2} \equiv D G M\left({ }_{3} \alpha,{ }_{3} \beta,{ }_{3} \gamma, 1\right)$, where $\left\{{ }_{3} \alpha\right\},\left\{{ }_{3} \beta\right\}$ and $\left\{{ }_{3} \gamma\right\}$ are the sequences defined by the formulas on the right sides of the inequalities (2.9), (2.10) and (2.11); respectively.

In this paper we shall present some properties of the classes $D G M\left({ }_{2} \alpha, 2 \beta, 2 \gamma, 2\right)$ and $D G M\left({ }_{3} \alpha,{ }_{3} \beta,{ }_{3} \gamma, 2\right)$. Moreover, we generalize and extend the results of Kórus ([4) to the classes $D G M\left({ }_{2} \alpha,,_{2} \beta,{ }_{2} \gamma, 2\right)$ or $D G M\left({ }_{3} \alpha,{ }_{3} \beta,{ }_{3} \gamma, 2\right)$, respectively.

\section{Auxiliary results}

Lemma 1. If $\left\{c_{j k}\right\} \subset \mathbb{C}$ is such that condition (2.2) and the inequality

$$
\sum_{j=m}^{2 m-1} \sum_{k=n}^{2 n-1}\left|\Delta_{22} c_{j k}\right| \leq \frac{\mathcal{C}}{m n}\left(\sup _{M+N \geq b(m+n)} \sum_{j=M}^{2 M} \sum_{k=N}^{2 N}\left|c_{j k}\right|\right), m, n \geq \lambda
$$

are satisfied, then

$$
m n \sum_{j=m}^{\infty} \sum_{k=n}^{\infty}\left|\Delta_{22} c_{j k}\right| \rightarrow 0 \quad \text { as } \quad m+n \rightarrow \infty \quad m, n \geq \lambda
$$

Proof. Set $\epsilon>0$ arbitrarily. By condition (2.2) and from the fact that $\{b(l)\}$ tends monotonically to infinity, there exists an $m_{1}=m_{1}(\epsilon)$ such that

$$
j k\left|c_{j k}\right|<\epsilon \quad \text { for all } j, k \quad j+k>b\left(m_{1}\right) .
$$

Then, by (3.1), assuming $m+n>m_{1}$ and $m, n \geq \lambda$, we have

$$
\begin{aligned}
\sum_{j=m}^{\infty} \sum_{k=n}^{\infty}\left|\Delta_{22} c_{j k}\right| & =\sum_{r=0}^{\infty} \sum_{s=0}^{\infty} \sum_{j=2^{r} m}^{2^{r+1} m-1} \sum_{k=2^{s} n}^{2^{s+1} n-1}\left|\Delta_{22} c_{j k}\right| \leq \sum_{r=0}^{\infty} \sum_{s=0}^{\infty} \frac{\mathcal{C}}{2^{r} m 2^{s} n}\left(\sup _{M+N \geq b\left(2^{r} m+2^{s} n\right)} \sum_{j=M}^{2 M} \sum_{k=N}^{2 N}\left|c_{j k}\right|\right) \\
& <\sum_{r=0}^{\infty} \sum_{s=0}^{\infty} \frac{\mathcal{C} \epsilon}{2^{r} m 2^{s} n}\left(\sup _{M+N \geq b\left(2^{r} m+2^{s} n\right)} \sum_{j=M}^{2 M} \sum_{k=N}^{2 N} \frac{1}{j k}\right) \leq \sum_{r=0}^{\infty} \sum_{s=0}^{\infty} \frac{4 \mathcal{C} \epsilon}{2^{r} m 2^{s} n} \leq \frac{16 \mathcal{C} \epsilon}{m n},
\end{aligned}
$$

since

$$
\sum_{j=M}^{2 M} \sum_{k=N}^{2 N} \frac{1}{j k} \leq \sum_{j=M}^{2 M} \sum_{k=N}^{2 N} \frac{1}{M N} \leq \frac{2 M \cdot 2 N}{M N}=4 \quad \text { for any } \quad M, N .
$$

This complete the proof. 
Lemma 2. Let $\left\{c_{j k}\right\}_{j, k=1}^{\infty} \subset \mathbb{C}$ and the condition 2.2) is satisfied.

(i) If the inequality

$$
\sum_{j=m}^{2 m-1}\left|\Delta_{20} c_{j n}\right| \leq \frac{\mathcal{C}}{m}\left(\sup _{M \geq b(m)} \sum_{j=M}^{2 M}\left|c_{j n}\right|\right), m \geq \lambda, n \geq 1
$$

holds, then

$$
m \sup _{k \geq n} k \sum_{j=m}^{\infty}\left|\Delta_{20} c_{j k}\right| \rightarrow 0
$$

(ii) and if the inequality

$$
\sum_{k=n}^{2 n-1}\left|\Delta_{02} c_{k m}\right| \leq \frac{\mathcal{C}}{n}\left(\sup _{N \geq b(n)} \sum_{k=N}^{2 N}\left|c_{k m}\right|\right), n \geq \lambda, m \geq 1
$$

holds, then

$$
n \sup _{j \geq m} j \sum_{k=n}^{\infty}\left|\Delta_{02} c_{j k}\right| \rightarrow 0
$$

as $m+n \rightarrow \infty$, where $m \geq \lambda$ and $n \geq 1$ or $n \geq \lambda$ and $m \geq 1$, respectively.

Proof. Set $\epsilon>0$ arbitrarily. By condition (2.2) and from the fact that $\{b(l)\}$ tends monotonically to infinity, there exists an $m_{1}=m_{1}(\epsilon)$ such that

$$
j k\left|c_{j k}\right|<\epsilon \quad \text { for all } j, k \quad j+k>b\left(m_{1}\right) .
$$

Part (i) By (3.3), assuming $m>\max \left\{m_{1}, \lambda\right\}$ and $n>m_{1}$, we have

$$
\begin{aligned}
\sup _{k \geq n} k \sum_{j=m}^{\infty}\left|\Delta_{20} c_{j k}\right| & =\sup _{k \geq n} k \sum_{r=0}^{\infty} \sum_{j=2^{r} m}^{2^{r+1} m-1}\left|\Delta_{20} c_{j k}\right| \leq \sup _{k \geq n} k \sum_{r=0}^{\infty} \frac{\mathcal{C}}{2^{r} m}\left(\sup _{M \geq b\left(2^{r} m\right)} \sum_{j=M}^{2 M}\left|c_{j k}\right|\right) \\
& \leq \sup _{k \geq n} k \frac{\mathcal{C}}{m} \sum_{r=0}^{\infty} \frac{1}{2^{r}}\left(\sup _{M \geq b\left(2^{r} m\right)} \sum_{j=M}^{2 M} j k\left|c_{j k}\right| \frac{1}{j k}\right)<\frac{\mathcal{C} \epsilon}{m} \sum_{r=0}^{\infty} \frac{1}{2^{r}}\left(\sup _{M \geq b\left(2^{r} m\right)} \sum_{j=M}^{2 M} \frac{1}{j}\right) \leq \frac{4 \mathcal{C} \epsilon}{m},
\end{aligned}
$$

since

$$
\sum_{j=M}^{2 M} \frac{1}{j} \leq \frac{M+1}{M} \leq 2
$$

This implies that (3.4) holds.

Part (ii) Using (3.5) and assuming $n>\max \left\{m_{1}, \lambda\right\}$ and $m>m_{1}$, we get

$$
\begin{aligned}
\sup _{m \geq j} j \sum_{k=n}^{\infty}\left|\Delta_{02} c_{j k}\right| & =\sup _{m \geq j} j \sum_{r=0}^{\infty} \sum_{k=2^{r} n}^{2^{r+1} n-1}\left|\Delta_{02} c_{j k}\right| \leq \sup _{m \geq j} j \sum_{r=0}^{\infty} \frac{\mathcal{C}}{2^{r} n}\left(\sup _{N \geq b\left(2^{r} n\right)} \sum_{k=N}^{2 N}\left|c_{j k}\right|\right) \\
& \leq \sup _{m \geq j} j \frac{\mathcal{C}}{n} \sum_{r=0}^{\infty} \frac{1}{2^{r}}\left(\sup _{N \geq b\left(2^{r} n\right)} \sum_{k=N}^{2 N} j k\left|c_{j k}\right| \frac{1}{j k}\right)<\frac{\mathcal{C} \epsilon}{n} \sum_{r=0}^{\infty} \frac{1}{2^{r}}\left(\sup _{N \geq b\left(2^{r} n\right)} \sum_{k=N}^{2 N} \frac{1}{k}\right) \leq \frac{4 \mathcal{C} \epsilon}{n} .
\end{aligned}
$$

Hence (3.6) is satisfied.

Now, our proof is complete.

Lemma 3. If $\left\{c_{j k}\right\}_{j, k=1}^{\infty}$ is a non-negative sequence belonging to the class $D G M\left({ }_{2} \alpha,{ }_{2} \beta,,_{2} \gamma, 2\right)$ with $\mathcal{C}, \lambda$ and $\left\{b_{1}(l)\right\}_{l=1}^{\infty},\left\{b_{2}(l)\right\}_{l=1}^{\infty},\left\{b_{3}(l)\right\}_{l=1}^{\infty}$ then for any $m, n \geq \lambda$

$$
m n c_{m n} \leq \mathcal{C}\left(\sup _{M+N \geq b_{3}(m+n)} \sum_{j=M}^{2 M} \sum_{k=N}^{2 N} c_{j k}\right)+2 \mathcal{C} \sum_{j=b_{1}(m)}^{2 \lambda b_{1}(m)} \sum_{k=n}^{2 n+1} c_{j k}+2 \mathcal{C} \sum_{j=m}^{2 m+1} \sum_{k=b_{2}(n)}^{2 \lambda b_{2}(n)} c_{j k}+8 \sum_{j=m}^{2 m+1} \sum_{k=n}^{2 n+1} c_{j k} .
$$


Proof. Let $m, n \geq \lambda$. If $\left\{c_{j k}\right\}_{j, k=1}^{\infty} \in D G M\left({ }_{2} \alpha,,_{2} \beta, 2 \gamma, 2\right)$, then for any $v$ and $m \leq \mu \leq 2 m$

$$
\begin{aligned}
c_{m v} & =\sum_{j=m}^{\mu-1} \Delta_{20} c_{j v}+c_{\mu v}+c_{\mu+1, v}-c_{m+1, v} \leq \sum_{j=m}^{2 m-1}\left|\Delta_{20} c_{j v}\right|+c_{\mu v}+c_{\mu+1, v} \\
& \leq \frac{\mathcal{C}}{m}\left(\max _{b_{1}(m) \leq M \leq \lambda b_{1}(m)} \sum_{j=M}^{2 M} c_{j v}\right)+c_{\mu v}+c_{\mu+1, v} .
\end{aligned}
$$

By an analogous argument, we get that for any $\mu$ and $n \leq v \leq 2 n$

$$
\begin{aligned}
c_{\mu n} & =\sum_{k=n}^{v-1} \Delta_{02} c_{\mu k}+c_{\mu v}+c_{\mu, v+1}-c_{\mu, n+1} \leq \sum_{k=n}^{2 n-1}\left|\Delta_{02} c_{\mu k}\right|+c_{\mu v}+c_{\mu, v+1} \\
& \leq \frac{\mathcal{C}}{n}\left(\max _{b_{2}(n) \leq N \leq \lambda b_{2}(n)} \sum_{k=N}^{2 N} c_{\mu k}\right)+c_{\mu v}+c_{\mu, v+1} .
\end{aligned}
$$

For $\mu, v$ such that $m \leq \mu \leq 2 m$ and $n \leq v \leq 2 n$ we have

$$
\begin{aligned}
\sum_{j=m}^{\mu-1} \sum_{k=n}^{v-1} \Delta_{22} c_{j k} & =\sum_{j=m}^{\mu-1} \sum_{k=n}^{v-1}\left(c_{j k}-c_{j+2, k}-\left(c_{j, k+2}-c_{j+2, k+2}\right)\right) \\
& =\sum_{k=n}^{v-1}\left(c_{m k}-c_{\mu k}-c_{\mu+1, k}+c_{m+1, k}-c_{m, k+2}+c_{\mu, k+2}+c_{\mu+1, k+2}-c_{m+1, k+2}\right) \\
& =\sum_{k=n}^{v-1}\left(\left(\left(c_{m k}-c_{m, k+2}\right)+\left(c_{m+1, k}-c_{m+1, k+2}\right)\right)-\left(\left(c_{\mu k}-c_{\mu, k+2}\right)+\left(c_{\mu+1, k}-c_{\mu+1, k+2}\right)\right)\right) \\
& =c_{m n}-c_{m v}-c_{m, v+1}+c_{m, n+1}+c_{m+1, n}-c_{m+1, v}-c_{m+1, v+1}+c_{m+1, n+1}-c_{\mu n} \\
& +c_{\mu v}+c_{\mu, v+1}-c_{\mu, n+1}-c_{\mu+1, n}+c_{\mu+1, v}+c_{\mu+1, v+1}-c_{\mu+1, n+1}
\end{aligned}
$$

and applying the inequality (3.1), we get

$$
\begin{aligned}
c_{m n} & =\sum_{j=m}^{\mu-1} \sum_{k=n}^{v-1} \Delta_{22} c_{j k}+c_{m v}+c_{m, v+1}-c_{m, n+1}-c_{m+1, n}+c_{m+1, v}+c_{m+1, v+1}-c_{m+1, n+1} \\
& +c_{\mu n}-c_{\mu v}-c_{\mu, v+1}+c_{\mu, n+1}+c_{\mu+1, n}-c_{\mu+1, v}-c_{\mu+1, v+1}+c_{\mu+1, n+1} \\
& \leq \sum_{j=m}^{2 m-1} \sum_{k=n}^{2 n-1}\left|\Delta_{22} c_{j k}\right|+c_{m v}+c_{m, v+1}+c_{m+1, v}+c_{m+1, v+1}+c_{\mu n}+c_{\mu, n+1}+c_{\mu+1, n}+c_{\mu+1, n+1} \\
& \leq \frac{\mathcal{C}}{m n}\left(\sup _{M+N \geq b_{3}(m+n)} \sum_{j=M}^{2 M} \sum_{k=N}^{2 N} c_{j k}\right) \\
& +c_{m v}+c_{m, v+1}+c_{m+1, v}+c_{m+1, v+1}+c_{\mu n}+c_{\mu, n+1}+c_{\mu+1, n}+c_{\mu+1, n+1} .
\end{aligned}
$$

Adding up all inequalities in (3.9) for $\mu=m+1, m+2, \ldots, 2 m$ and $v=n+1, n+2, \ldots, 2 n$ we obtain

$$
\begin{aligned}
\sum_{\mu=m+1}^{2 m} \sum_{v=n+1}^{2 n} c_{m n} & \leq \sum_{\mu=m+1}^{2 m} \sum_{v=n+1}^{2 n} \frac{\mathcal{C}}{m n}\left(\sup _{M+N \geq b_{3}(m+n)} \sum_{j=M}^{2 M} \sum_{k=N}^{2 N} c_{j k}\right) \\
& +\sum_{\mu=m+1}^{2 m} \sum_{v=n+1}^{2 n} c_{m v}+\sum_{\mu=m+1}^{2 m} \sum_{v=n+1}^{2 n} c_{m, v+1}+\sum_{\mu=m+1}^{2 m} \sum_{v=n+1}^{2 n} c_{m+1, v}+\sum_{\mu=m+1}^{2 m} \sum_{v=n+1}^{2 n} c_{m+1, v+1} \\
& +\sum_{v=n+1}^{2 n} \sum_{\mu=m+1}^{2 m} c_{\mu n}+\sum_{v=n+1}^{2 n} \sum_{\mu=m+1}^{2 m} c_{\mu, n+1}+\sum_{v=n+1}^{2 n} \sum_{\mu=m+1}^{2 m} c_{\mu+1, n}+\sum_{v=n+1}^{2 n} \sum_{\mu=m+1}^{2 m} c_{\mu+1, n+1}
\end{aligned}
$$




$$
\begin{aligned}
& =\mathcal{C}\left(\sup _{M+N \geq b_{3}(m+n)} \sum_{j=M}^{2 M} \sum_{k=N}^{2 N} c_{j k}\right)+\sum_{\mu=m+1}^{2 m} \sum_{v=n+1}^{2 n}\left(c_{m v}+c_{m+1, v}\right)+\sum_{\mu=m+1}^{2 m} \sum_{v=n+1}^{2 n}\left(c_{m, v+1}+c_{m+1, v+1}\right) \\
& +\sum_{v=n+1}^{2 n} \sum_{\mu=m+1}^{2 m}\left(c_{\mu n}+c_{\mu, n+1}\right)+\sum_{v=n+1}^{2 n} \sum_{\mu=m+1}^{2 m}\left(c_{\mu+1, n}+c_{\mu+1, n+1}\right) .
\end{aligned}
$$

Analogously as in (3.7) and (3.8) we can obtain the following inequalities

$$
\begin{aligned}
c_{m v}+c_{m+1, v} \leq \frac{\mathcal{C}}{m}\left(\max _{b_{1}(m) \leq M \leq \lambda b_{1}(m)} \sum_{j=M}^{2 M} c_{j v}\right)+c_{\mu v}+c_{\mu+1, v} \quad \text { for any } v \in \mathbb{N} \text { and } \mu=m, \cdots, 2 m, \\
c_{\mu n}+c_{\mu, n+1} \leq \frac{\mathcal{C}}{n}\left(\max _{b_{2}(n) \leq N \leq \lambda b_{2}(n)} \sum_{k=N}^{2 N} c_{\mu k}\right)+c_{\mu v}+c_{\mu, v+1} \quad \text { for any } \mu \in \mathbb{N} \text { and } v=n, \cdots, 2 n .
\end{aligned}
$$

Hence we get

$$
\begin{aligned}
& m n c_{m n} \leq \mathcal{C}\left(\sup _{M+N \geq b_{3}(m+n)} \sum_{j=M}^{2 M} \sum_{k=N}^{2 N} c_{j k}\right) \\
& +\sum_{v=n+1}^{2 n}\left(\sum_{\mu=m+1}^{2 m} \frac{\mathcal{C}}{m}\left(\max _{b_{1}(m) \leq M \leq \lambda b_{1}(m)} \sum_{j=M}^{2 M} c_{j v}\right)+\sum_{\mu=m+1}^{2 m}\left(c_{\mu v}+c_{\mu+1, v}\right)\right) \\
& +\sum_{v=n+1}^{2 n}\left(\sum_{\mu=m+1}^{2 m} \frac{\mathcal{C}}{m}\left(\max _{b_{1}(m) \leq M \leq \lambda b_{1}(m)} \sum_{j=M}^{2 M} c_{j, v+1}\right)+\sum_{\mu=m+1}^{2 m}\left(c_{\mu, v+1}+c_{\mu+1, v+1}\right)\right) \\
& +\sum_{\mu=m+1}^{2 m}\left(\sum_{v=n+1}^{2 n} \frac{\mathcal{C}}{n}\left(\max _{b_{2}(n) \leq N \leq \lambda b_{2}(n)} \sum_{k=N}^{2 N} c_{\mu k}\right)+\sum_{v=n+1}^{2 n}\left(c_{\mu v}+c_{\mu, v+1}\right)\right) \\
& +\sum_{\mu=m+1}^{2 m}\left(\sum_{v=n+1}^{2 n} \frac{\mathcal{C}}{n}\left(\max _{b_{2}(n) \leq N \leq \lambda b_{2}(n)} \sum_{k=N}^{2 N} c_{\mu+1, k}\right)+\sum_{v=n+1}^{2 n}\left(c_{\mu+1, v}+c_{\mu+1, v+1}\right)\right) \\
& \leq \mathcal{C}\left(\sup _{M+N \geq b_{3}(m+n)} \sum_{j=M}^{2 M} \sum_{k=N}^{2 N} c_{j k}\right)+\mathcal{C} \sum_{j=b_{1}(m)}^{2 \lambda b_{1}(m)} \sum_{k=n}^{2 n} c_{j k}+\sum_{j=m}^{2 m} \sum_{k=n}^{2 n} c_{j k}+\sum_{j=m}^{2 m} \sum_{k=n}^{2 n} c_{j+1, k} \\
& +\mathcal{C} \sum_{j=b_{1}(m)}^{2 \lambda b_{1}(m)} \sum_{k=n}^{2 n} c_{j, k+1}+\sum_{j=m}^{2 m} \sum_{k=n}^{2 n} c_{j, k+1}+\sum_{j=m}^{2 m} \sum_{k=n}^{2 n} c_{j+1, k+1}+ \\
& +\mathcal{C} \sum_{j=m}^{2 m} \sum_{k=b_{2}(n)}^{2 \lambda b_{2}(n)} c_{j k}+\sum_{j=m}^{2 m} \sum_{k=n}^{2 n} c_{j k}+\sum_{j=m}^{2 m} \sum_{k=n}^{2 n} c_{j, k+1} \\
& +\mathcal{C} \sum_{j=m}^{2 m} \sum_{k=b_{2}(n)}^{2 \lambda b_{2}(n)} c_{j+1, k}+\sum_{j=m}^{2 m} \sum_{k=n}^{2 n} c_{j+1, k}+\sum_{j=m}^{2 m} \sum_{k=n}^{2 n} c_{j+1, k+1} \\
& \leq \mathcal{C}\left(\sup _{M+N \geq b_{3}(m+n)} \sum_{j=M}^{2 M} \sum_{k=N}^{2 N} c_{j k}\right)+2 \mathcal{C} \sum_{j=b_{1}(m)}^{2 \lambda b_{1}(m)} \sum_{k=n}^{2 n+1} c_{j k}+2 \mathcal{C} \sum_{j=m}^{2 m+1} \sum_{k=b_{2}(n)}^{2 \lambda b_{2}(n)} c_{j k}+8 \sum_{j=m}^{2 m+1} \sum_{k=n}^{2 n+1} c_{j k} .
\end{aligned}
$$

This ends the proof.

Denote, for $r \in \mathbb{N}$ and $k=0,1,2, \ldots$, by

$$
\widetilde{D}_{k, r}(x)=\frac{\cos \left(k+\frac{r}{2}\right) x}{2 \sin \left(\frac{r}{2} x\right)}
$$

the conjugated Dirichlet type kernel. 
Lemma 4. ([6], [7]) Let $r \in \mathbb{N}, l \in \mathbb{Z}$ and $\left\{a_{k}\right\}_{k=1}^{\infty} \subset \mathbb{C}$. If $x \neq \frac{2 l \pi}{r}$, then for all $m \geq n$

$$
\sum_{k=n}^{m} a_{k} \sin k x=-\sum_{k=n}^{m} \Delta_{r} a_{k} \widetilde{D}_{k, r}(x)+\sum_{k=m+1}^{m+r} a_{k} \widetilde{D}_{k,-r}(x)-\sum_{k=n}^{n+r-1} a_{k} \widetilde{D}_{k,-r}(x)
$$

Lemma 5. Let $\left\{c_{j k}\right\}_{j, k=1}^{\infty} \subset \mathbb{C}$ and $m, M, n, N \in \mathbb{N}$ such that $m \leq M$ and $n \leq N$.

(i) If $x \in\left(0, \frac{\pi}{2}\right)$, then

$$
\left|\sum_{j=m}^{M} c_{j k} \sin j x\right| \leq \frac{\pi}{4 x}\left(\sum_{j=m}^{M}\left|\Delta_{20} c_{j k}\right|+\sum_{j=M+1}^{M+2}\left|c_{j k}\right|+\sum_{j=m}^{m+1}\left|c_{j k}\right|\right)
$$

and if $x \in\left(\frac{\pi}{2}, \pi\right)$, then

$$
\left|\sum_{j=m}^{M} c_{j k} \sin j x\right| \leq \frac{\pi}{4(\pi-x)}\left(\sum_{j=m}^{M}\left|\Delta_{20} c_{j k}\right|+\sum_{j=M+1}^{M+2}\left|c_{j k}\right|+\sum_{j=m}^{m+1}\left|c_{j k}\right|\right)
$$

for any $k \in \mathbb{N}$.

(ii) If $y \in\left(0, \frac{\pi}{2}\right)$, then

$$
\left|\sum_{k=n}^{N} c_{j k} \sin k y\right| \leq \frac{\pi}{4 y}\left(\sum_{k=n}^{N}\left|\Delta_{02} c_{j k}\right|+\sum_{k=N+1}^{N+2}\left|c_{j k}\right|+\sum_{k=n}^{n+1}\left|c_{j k}\right|\right)
$$

and if $y \in\left(\frac{\pi}{2}, \pi\right)$, then

$$
\left|\sum_{k=n}^{N} c_{j k} \sin k y\right| \leq \frac{\pi}{4(\pi-y)}\left(\sum_{k=n}^{N}\left|\Delta_{02} c_{j k}\right|+\sum_{k=N+1}^{N+2}\left|c_{j k}\right|+\sum_{k=n}^{n+1}\left|c_{j k}\right|\right)
$$

for any $j \in \mathbb{N}$.

Proof. Part (i). By Lemma 4, we have

$$
\begin{aligned}
\left|\sum_{j=m}^{M} c_{j k} \sin j x\right| & =\left|-\sum_{j=m}^{M} \Delta_{20} c_{j k} \widetilde{D}_{j, 2}(x)+\sum_{j=M+1}^{M+2} c_{j k} \widetilde{D}_{j,-2}(x)-\sum_{j=m}^{m+1} c_{j k} \widetilde{D}_{j,-2}(x)\right| \\
& \leq \sum_{j=m}^{M}\left|\Delta_{20} c_{j k}\right| \cdot\left|\widetilde{D}_{j, 2}(x)\right|+\sum_{j=M+1}^{M+2}\left|c_{j k}\right| \cdot\left|\widetilde{D}_{j,-2}(x)\right|+\sum_{j=m}^{m+1}\left|c_{j k}\right| \cdot\left|\widetilde{D}_{j,-2}(x)\right| .
\end{aligned}
$$

If $x \in\left(0, \frac{\pi}{2}\right)$, then using inequality $\sin x \geq \frac{2}{\pi} x$ we obtain the following estimation:

$$
\left|\widetilde{D}_{j, \pm 2}(x)\right| \leq\left|\frac{\cos (j \pm 1) x}{2 \sin ( \pm x)}\right| \leq \frac{1}{2 \sin x} \leq \frac{1}{\frac{4}{\pi} x} \leq \frac{\pi}{4 x} .
$$

From this we get

$$
\left|\sum_{j=m}^{M} c_{j k} \sin j x\right| \leq \frac{\pi}{4 x}\left(\sum_{j=m}^{M}\left|\Delta_{20} c_{j k}\right|+\sum_{j=M+1}^{M+2}\left|c_{j k}\right|+\sum_{j=m}^{m+1}\left|c_{j k}\right|\right) .
$$

If $x \in\left(\frac{\pi}{2}, \pi\right)$, then using inequality $\sin x \geq 2-\frac{2}{\pi} x$ we have the estimation:

$$
\left|\widetilde{D}_{j, \pm 2}(x)\right| \leq \frac{1}{2\left(2-\frac{2}{\pi} x\right)} \leq \frac{\pi}{4(\pi-x)}
$$

and consequently

$$
\left|\sum_{j=m}^{M} c_{j k} \sin j x\right| \leq \frac{\pi}{4(\pi-x)}\left(\sum_{j=m}^{M}\left|\Delta_{20} c_{j k}\right|+\sum_{j=M+1}^{M+2}\left|c_{j k}\right|+\sum_{j=m}^{m+1}\left|c_{j k}\right|\right) .
$$


Part (ii). Analogously as above

$$
\begin{aligned}
\left|\sum_{k=n}^{N} c_{j k} \sin k y\right| & =\left|-\sum_{k=n}^{N} \Delta_{02} c_{j k} \widetilde{D}_{k, 2}(y)+\sum_{k=N+1}^{N+2} c_{j k} \widetilde{D}_{k,-2}(y)-\sum_{k=n}^{n+1} c_{j k} \widetilde{D}_{k,-2}(y)\right| \\
& \leq \sum_{k=n}^{N}\left|\Delta_{02} c_{j k}\right| \cdot\left|\widetilde{D}_{k, 2}(y)\right|+\sum_{k=N+1}^{N+2}\left|c_{j k}\right| \cdot\left|\widetilde{D}_{k,-2}(y)\right|+\sum_{k=n}^{n+1}\left|c_{j k}\right| \cdot\left|\widetilde{D}_{k,-2}(y)\right| \\
& \leq \frac{\pi}{4 y}\left(\sum_{k=n}^{N}\left|\Delta_{02} c_{j k}\right|+\sum_{k=N+1}^{N+2}\left|c_{j k}\right|+\sum_{k=n}^{n+1}\left|c_{j k}\right|\right)
\end{aligned}
$$

for $y \in\left(0, \frac{\pi}{2}\right)$, and

$$
\left|\sum_{k=n}^{N} c_{j k} \sin k y\right| \leq \frac{\pi}{4(\pi-y)}\left(\sum_{k=n}^{N}\left|\Delta_{02} c_{j k}\right|+\sum_{k=N+1}^{N+2}\left|c_{j k}\right|+\sum_{k=n}^{n+1}\left|c_{j k}\right|\right)
$$

for $y \in\left(\frac{\pi}{2}, \pi\right)$.

This ends the proof.

\section{Main results}

We have the following results:

\section{Theorem 7.}

(i) If a double sequence $\left\{c_{j k}\right\}_{j, k=1}^{\infty} \subset \mathbb{C}$ belongs to $D G M\left({ }_{3} \alpha,{ }_{3} \beta, 3 \gamma, 2\right)$ and (2.2) holds, then the regular convergence of double sine series (2.1) is uniform in $(x, y)$.

(ii) Conversely, if a double sequence $\left\{c_{j k}\right\}_{j, k=1}^{\infty} \subset \mathbb{R}_{+}$belongs to $D G M\left({ }_{2} \alpha, 2 \beta, 2 \gamma, 2\right)$ and double sine series (2.1) is uniformly regularly convergent in $(x, y)$, then (2.2) is satisfied.

\section{Theorem 8.}

(i) $\operatorname{DGM}\left({ }_{3} \alpha,_{3} \beta,,_{3} \gamma, 1\right) \subset D G M\left({ }_{3} \alpha,{ }_{3} \beta,{ }_{3} \gamma, 2\right)$.

(ii) There exists a double sequence $\left\{c_{j k}\right\}_{j, k=1}^{\infty}$, with the property (2.2), which belongs to the class $D G M\left({ }_{3} \alpha,_{3} \beta,{ }_{3} \gamma, 2\right)$ but it does not belong to the class DGM $\left({ }_{3} \alpha,_{3} \beta,{ }_{3} \gamma, 1\right)$.

Analogously as in Theorem 8, we can show:

\section{Corollary 1.}

(i) $\operatorname{DGM}\left({ }_{2} \alpha,{ }_{2} \beta,{ }_{2} \gamma, 1\right) \subset \operatorname{DGM}\left({ }_{2} \alpha,{ }_{2} \beta,{ }_{2} \gamma, 2\right)$.

(ii) There exists a double sequence $\left\{c_{j k}\right\}_{j, k=1}^{\infty}$, with the property (2.2), which belongs to the class $D G M\left({ }_{2} \alpha,{ }_{2} \beta,{ }_{2} \gamma, 2\right)$ but it does not belong to the class DGM $\left({ }_{2} \alpha,{ }_{2} \beta,{ }_{2} \gamma, 1\right)$.

Now, we formulate some remarks.

Remark 1. From Theorem 7 , using Theorem 8 and Corollary 1, we obtain Theorem 5 and Theorem 4.

Remark 2. There exist $\left(x_{0}, y_{0}\right) \in \mathbb{R}^{2}$ and a sequence $\left\{c_{j k}\right\}_{j, k=1}^{\infty}$ belonging to the class DGM $\left({ }_{3} \alpha,{ }_{3} \beta,,_{3} \gamma, 3\right)$, with the property (2.2), such that the series (2.1) is divergent in $\left(x_{0}, y_{0}\right)$.

Remark 3. Remark 圆 shows that the results from Theorem 7 are not true with $r=3$ instead of $r=2$. 


\section{Proof of the main results}

In this section we shall prove our main results.

\subsection{Proof of Theorem 7}

Part (i): Analogously as in ([7], Theorem 2.5) we can show that single series:

$$
\sum_{j=1}^{\infty} c_{j n} \sin j x, \quad n=1,2, \ldots, \quad \sum_{k=1}^{\infty} c_{m k} \sin k y, \quad m=1,2, \ldots
$$

are uniformly convergent since $\left\{c_{j n}\right\}_{j=1}^{\infty} \in G M\left({ }_{3} \alpha, 2\right)$ for any $n \in \mathbb{N}$ and $\left\{c_{m k}\right\}_{k=1}^{\infty} \in G M\left({ }_{3} \beta, 2\right)$ for any $m \in \mathbb{N}$. Let $\epsilon>0$ be arbitrarily fixed. We shall prove that for any $M \geq m>\eta, N \geq n>\eta$ and any $(x, y) \in \mathbb{R}^{2}$ we have

$$
\left|\sum_{j=m}^{M} \sum_{k=n}^{N} c_{j k} \sin j x \sin k y\right|<\left(1+2 \pi \mathcal{C}+2 \pi+1,5 \pi^{2} \mathcal{C}+\pi^{2}\right) \epsilon,
$$

where $\eta=\eta(\epsilon)>\lambda$ is the natural number which satisfies for any $m, n>\eta$

$m n\left|c_{m n}\right|<\epsilon, \quad m n \sum_{j=m}^{\infty} \sum_{k=n}^{\infty}\left|\Delta_{22} c_{j k}\right|<16 C \epsilon, \quad m \sum_{j=m}^{\infty} \sup _{k \geq n} k\left|\Delta_{20} c_{j k}\right|<4 C \epsilon, \quad n \sum_{k=n}^{\infty} \sup _{j \geq m} j\left|\Delta_{02} c_{j k}\right|<4 C \epsilon$.

The inequality (5.2) is trivial, when $x=0$ and $y$ is arbitrary or $y=0$ and $x$ is arbitrary. We have the same situation, if $x=\pi$ and $y$ is arbitrary or $y=\pi$ and $x$ is arbitrary. Suppose $x, y \in\left(0, \frac{\pi}{2}\right)$, set $\mu:=\left\lceil\frac{1}{x}\right\rceil$ and $v:=\left\lceil\frac{1}{y}\right\rceil$, where $\lceil\cdot\rceil$ means the integer part of a real number. We have four cases:

CASE (a): $\eta<m \leq M<\mu$ and $\eta<n \leq N<v$. Using the inequality $\sin x \leq x$, we have

$$
\left|\sum_{j=m}^{M} \sum_{k=n}^{N} c_{j k} \sin j x \sin k y\right| \leq x y \sum_{j=m}^{M} \sum_{k=n}^{N} j k\left|c_{j k}\right|<\frac{1}{\mu v} \sum_{j=m}^{\mu} \sum_{k=n}^{v} \epsilon \leq \epsilon .
$$

CASE (b): $\max \{\eta, \mu\}<m \leq M$ and $\eta<n \leq N<v$. We obtain

$$
\left|\sum_{j=m}^{M} \sum_{k=n}^{N} c_{j k} \sin j x \sin k y\right| \leq \sum_{k=n}^{N}|\sin k y| \cdot\left|\sum_{j=m}^{M} c_{j k} \sin j x\right| \leq y \sum_{k=n}^{N} k\left|\sum_{j=m}^{M} c_{j k} \sin j x\right| .
$$

By $(3.10)$

$$
\begin{aligned}
\left|\sum_{j=m}^{M} \sum_{k=n}^{N} c_{j k} \sin j x \sin k y\right| & \leq y \sum_{k=n}^{N} k \frac{\pi}{4 x}\left(\sum_{j=m}^{M}\left|\Delta_{20} c_{j k}\right|+\sum_{j=M+1}^{M+2}\left|c_{j k}\right|+\sum_{j=m}^{m+1}\left|c_{j k}\right|\right) \\
& \leq y \sum_{k=n}^{N} k \frac{\pi \mu}{4}\left(\sum_{j=m}^{M}\left|\Delta_{20} c_{j k}\right|+\sum_{j=M+1}^{M+2}\left|c_{j k}\right|+\sum_{j=m}^{m+1}\left|c_{j k}\right|\right) \\
& \leq \frac{\pi}{4 v} \sum_{k=n}^{v}\left(m \sup _{k \geq n} k \sum_{j=m}^{\infty}\left|\Delta_{20} c_{j k}\right|+4 \sup _{j \geq m} \sup _{k \geq n} j k\left|c_{j k}\right|\right)
\end{aligned}
$$

and using Lemma 2 and (2.2) we get

$$
\left|\sum_{j=m}^{M} \sum_{k=n}^{N} c_{j k} \sin j x \sin k y\right|<\frac{\pi}{4}(4 \mathcal{C} \epsilon+4 \epsilon)=(\pi \mathcal{C}+\pi) \epsilon
$$


CASE (c): $\eta<m \leq M<\mu$ and $\max \{\eta, v\}<n \leq N$. We have

$$
\left|\sum_{j=m}^{M} \sum_{k=n}^{N} c_{j k} \sin j x \sin k y\right| \leq \sum_{j=m}^{M}|\sin j x| \cdot\left|\sum_{k=n}^{N} c_{j k} \sin k y\right| \leq x \sum_{j=m}^{M} j\left|\sum_{k=n}^{N} c_{j k} \sin k y\right| .
$$

\section{Using (3.11)}

$$
\begin{aligned}
& \left|\sum_{j=m}^{M} \sum_{k=n}^{N} c_{j k} \sin j x \sin k y\right| \leq x \sum_{j=m}^{M} j \frac{\pi}{4 y}\left(\sum_{k=n}^{N}\left|\Delta_{02} c_{j k}\right|+\sum_{k=N+1}^{N+2}\left|c_{j k}\right|+\sum_{k=n}^{n+1}\left|c_{j k}\right|\right) \\
& \leq x \sum_{j=m}^{M} j \frac{\pi v}{4}\left(\sum_{k=n}^{N}\left|\Delta_{02} c_{j k}\right|+\sum_{k=N+1}^{N+2}\left|c_{j k}\right|+\sum_{k=n}^{n+1}\left|c_{j k}\right|\right) \\
& \leq \frac{\pi}{4 \mu} \sum_{j=m}^{\mu}\left(n \sup _{j \geq m} j \sum_{k=n}^{\infty}\left|\Delta_{02} c_{j k}\right|+4 \sup _{j \geq m k \geq n} j k\left|c_{j k}\right|\right)
\end{aligned}
$$

and by Lemma 2 and (2.2)

$$
\left|\sum_{j=m}^{M} \sum_{k=n}^{N} c_{j k} \sin j x \sin k y\right|<\frac{\pi}{4}(4 \mathcal{C} \epsilon+4 \epsilon)=(\pi \mathcal{C}+\pi) \epsilon .
$$

CASE (d): $\max \{\eta, \mu\}<m \leq M$ and $\max \{\eta, v\}<n \leq N$. Using Lemma 4 we get

$$
\begin{aligned}
\mid \sum_{j=m}^{M} \sum_{k=n}^{N} & c_{j k} \sin j x \sin k y|\leq| \sum_{j=m}^{M}\left(-\sum_{k=n}^{N} \Delta_{02} c_{j k} \widetilde{D}_{k, 2}(y)+\sum_{k=N+1}^{N+2} c_{j k} \widetilde{D}_{k,-2}(y)-\sum_{k=n}^{n+1} c_{j k} \widetilde{D}_{k,-2}(y)\right) \sin j x \mid \\
& =\mid-\sum_{k=n}^{N}\left(\sum_{j=m}^{M} \Delta_{02} c_{j k} \sin j x\right) \widetilde{D}_{k, 2}(y)+\sum_{k=N+1}^{N+2}\left(\sum_{j=m}^{M} c_{j k} \sin j x\right) \widetilde{D}_{k,-2}(y) \\
& -\sum_{k=n}^{n+1}\left(\sum_{j=m}^{M} c_{j k} \sin j x\right) \widetilde{D}_{k,-2}(y) \mid \\
& =\mid-\sum_{k=n}^{N}\left(-\sum_{j=m}^{M} \Delta_{02}\left(\Delta_{20} c_{j k}\right) \widetilde{D}_{j, 2}(x)+\sum_{j=M+1}^{M+2} \Delta_{02} c_{j k} \widetilde{D}_{j,-2}-\sum_{j=m}^{m+1} \Delta_{02} c_{j k} \widetilde{D}_{j,-2}(x)\right) \widetilde{D}_{k, 2}(y) \\
& +\sum_{k=N+1}^{N+2}\left(-\sum_{j=m}^{M} \Delta_{20} c_{j k} \widetilde{D}_{j, 2}(x)+\sum_{j=M+1}^{M+2} c_{j k} \widetilde{D}_{j,-2}-\sum_{j=m}^{m+1} c_{j k} \widetilde{D}_{j,-2}(x)\right) \widetilde{D}_{k,-2}(y) \\
& -\sum_{k=n+1}^{n+1}\left(-\sum_{j=m}^{M} \Delta_{20} c_{j k} \widetilde{D}_{j, 2}(x)+\sum_{j=M+1}^{M+2} c_{j k} \widetilde{D}_{j,-2}-\sum_{j=m}^{m+1} c_{j k} \widetilde{D}_{j,-2}(x)\right) \widetilde{D}_{k,-2}(y) \mid \\
& \leq \sum_{j=m}^{M} \sum_{k=n}^{N}\left|\Delta_{22} c_{j k}\right| \cdot\left|\widetilde{D}_{j, 2}(x)\right| \cdot\left|\widetilde{D}_{k, 2}(y)\right|+\sum_{j=M+1}^{M+2} \sum_{k=n}^{N}\left|\Delta_{02} c_{j k}\right| \cdot\left|\widetilde{D}_{j,-2}(x)\right| \cdot\left|\widetilde{D}_{k, 2}(y)\right| \\
& +\sum_{j=m}^{m+1} \sum_{k=n}^{N}\left|\Delta_{02} c_{j k}\right| \cdot\left|\widetilde{D}_{j,-2}(x)\right| \cdot\left|\widetilde{D}_{k, 2}(y)\right|+\sum_{j=m}^{M} \sum_{k=N+1}^{N+2}\left|\Delta_{20} c_{j k}\right| \cdot\left|\widetilde{D}_{j, 2}(x)\right| \cdot\left|\widetilde{D}_{k,-2}(y)\right| \\
& +\sum_{j=M+1}^{M+2} \sum_{k=N+1}^{N+2}\left|c_{j k}\right| \cdot\left|\widetilde{D}_{j,-2}(x)\right| \cdot\left|\widetilde{D}_{k,-2}(y)\right|+\sum_{j=m}^{m+1} \sum_{k=N+1}^{N+2}\left|c_{j k}\right| \cdot\left|\widetilde{D}_{j,-2}(x)\right| \cdot\left|\widetilde{D}_{k,-2}(y)\right|
\end{aligned}
$$




$$
\begin{aligned}
& +\sum_{j=m}^{M} \sum_{k=n}^{n+1}\left|\Delta_{20} c_{j k}\right| \cdot\left|\widetilde{D}_{j, 2}(x)\right| \cdot\left|\widetilde{D}_{k,-2}(y)\right|+\sum_{j=M+1}^{M+2} \sum_{k=n}^{n+1}\left|c_{j k}\right| \cdot\left|\widetilde{D}_{j,-2}(x)\right| \cdot\left|\widetilde{D}_{k,-2}(y)\right| \\
& +\sum_{j=m}^{m+1} \sum_{k=n}^{n+1}\left|c_{j k}\right| \cdot\left|\widetilde{D}_{j,-2}(x)\right| \cdot\left|\widetilde{D}_{k,-2}(y)\right| .
\end{aligned}
$$

Using (3.12), (2.2) Lemma 1 and Lemma 2, we obtain

$$
\begin{aligned}
\mid \sum_{j=m}^{M} & \sum_{k=n}^{N} c_{j k} \sin j x \sin k y \mid \leq \\
\leq & \frac{\pi^{2}}{16 x y}\left(\sum_{j=m}^{M} \sum_{k=n}^{N}\left|\Delta_{22} c_{j k}\right|+\sum_{j=M+1}^{M+2} \sum_{k=n}^{N}\left|\Delta_{02} c_{j k}\right|+\sum_{j=m}^{m+1} \sum_{k=n}^{N}\left|\Delta_{02} c_{j k}\right|+\sum_{j=m}^{M} \sum_{k=N+1}^{N+2}\left|\Delta_{20} c_{j k}\right|\right. \\
& \left.+\sum_{j=M+1}^{M+2} \sum_{k=N+1}^{N+2}\left|c_{j k}\right|+\sum_{j=m}^{m+1} \sum_{k=N+1}^{N+2}\left|c_{j k}\right|+\sum_{j=m}^{M} \sum_{k=n}^{n+1}\left|\Delta_{20} c_{j k}\right|+\sum_{j=M+1}^{M+2} \sum_{k=n}^{n+1}\left|c_{j k}\right|+\sum_{j=m}^{m+1} \sum_{k=n}^{n+1}\left|c_{j k}\right|\right) \\
& \leq \frac{\pi^{2}}{16}\left(m n \sum_{j=m}^{\infty} \sum_{k=n}^{\infty}\left|\Delta_{22} c_{j k}\right|+4 m \sup _{k \geq n} k \sum_{j=m}^{\infty}\left|\Delta_{20} c_{j k}\right|+4 n \sup _{j \geq m} j \sum_{k=n}^{\infty}\left|\Delta_{02} c_{j k}\right|+16 \sup _{j \geq m} \sup _{k \geq n} j k\left|c_{j k}\right|\right) \\
& <\frac{\pi^{2}}{16}(16 \mathcal{C} \epsilon+4 \mathcal{C} \epsilon+4 \mathcal{C} \epsilon+16 \epsilon)=\left(\frac{3}{2} \mathcal{C}+1\right) \pi^{2} \epsilon .
\end{aligned}
$$

Let $x \in\left(\frac{\pi}{2}, \pi\right)$ and $y \in\left(0, \frac{\pi}{2}\right)$, set $\mu:=\left\lceil\frac{1}{\pi-x}\right\rceil$ and $v:=\left\lceil\frac{1}{y}\right\rceil$. We have four cases:

CASE $\left(a^{*}\right): \eta<m \leq M<\mu$ and $\eta<n \leq N<v$. Using the inequality $\sin x \leq \pi-x$ and $\sin y \leq y$, we get

$$
\left|\sum_{j=m}^{M} \sum_{k=n}^{N} c_{j k} \sin j x \sin k y\right| \leq(\pi-x) y \sum_{j=m}^{M} \sum_{k=n}^{N} j k\left|c_{j k}\right|<\frac{1}{\mu v} \sum_{j=m}^{\mu} \sum_{k=n}^{v} \epsilon \leq \epsilon .
$$

CASE $\left(b^{*}\right): \max \{\eta, \mu\}<m \leq M$ and $\eta<n \leq N<v$. Applying (3.13) we get

$$
\begin{aligned}
\left|\sum_{j=m}^{M} \sum_{k=n}^{N} c_{j k} \sin j x \sin k y\right| & \leq \sum_{k=n}^{N}|\sin k y| \cdot\left|\sum_{j=m}^{M} c_{j k} \sin j x\right| \leq y \sum_{k=n}^{N} k\left|\sum_{j=m}^{M} c_{j k} \sin j x\right| \\
& \leq y \sum_{k=n}^{N} k \frac{\pi}{4(\pi-x)}\left(\sum_{j=m}^{M}\left|\Delta_{20} c_{j k}\right|+\sum_{j=M+1}^{M+2}\left|c_{j k}\right|+\sum_{j=m}^{m+1}\left|c_{j k}\right|\right)<(\pi \mathcal{C}+\pi) \epsilon .
\end{aligned}
$$

CASE $\left(c^{*}\right): \eta<m \leq M<\mu$ and $\max \{\eta, v\}<n \leq N$. Analogously as in case (c), we have the inequality

$$
\left|\sum_{j=m}^{M} \sum_{k=n}^{N} c_{j k} \sin j x \sin k y\right| \leq \sum_{j=m}^{M}|\sin j x| \cdot\left|\sum_{k=n}^{N} c_{j k} \sin k y\right| \leq(\pi-x) \sum_{j=m}^{M} j\left|\sum_{k=n}^{N} c_{j k} \sin k y\right|<(\pi \mathcal{C}+\pi) \epsilon .
$$


CASE $\left(d^{*}\right): \max \{\eta, \mu\}<m \leq M$ and $\max \{\eta, v\}<n \leq N$. Using (3.12) and (3.13) analogously as in case $(d)$, we obtain

$$
\begin{aligned}
& \mid \sum_{j=m}^{M} \sum_{k=n}^{N} c_{j k} \sin j x \sin k y \mid \leq \\
& \quad \leq \frac{\pi^{2}}{16(\pi-x) y}\left(\sum_{j=m}^{M} \sum_{k=n}^{N}\left|\Delta_{22} c_{j k}\right|+\sum_{j=M+1}^{M+2} \sum_{k=n}^{N}\left|\Delta_{02} c_{j k}\right|+\sum_{j=m}^{m+1} \sum_{k=n}^{N}\left|\Delta_{02} c_{j k}\right|+\sum_{j=m}^{M} \sum_{k=N+1}^{N+2}\left|\Delta_{20} c_{j k}\right|\right. \\
&\left.\quad+\sum_{j=M+1}^{M+2} \sum_{k=N+1}^{N+2}\left|c_{j k}\right|+\sum_{j=m}^{m+1} \sum_{k=N+1}^{N+2}\left|c_{j k}\right|+\sum_{j=m}^{M} \sum_{k=n}^{n+1}\left|\Delta_{20} c_{j k}\right|+\sum_{j=M+1}^{M+2} \sum_{k=n}^{n+1}\left|c_{j k}\right|+\sum_{j=m}^{m+1} \sum_{k=n}^{n+1}\left|c_{j k}\right|\right) \\
& \quad<\left(\frac{3}{2} \mathcal{C}+1\right) \pi^{2} \epsilon .
\end{aligned}
$$

Let $x \in\left(0, \frac{\pi}{2}\right)$ and $y \in\left(\frac{\pi}{2}, \pi\right)$, set $\mu:=\left\lceil\frac{1}{x}\right\rceil$ and $v:=\left\lceil\frac{1}{\pi-y}\right\rceil$. Now, we have also four cases:

CASE $\left(a^{* *}\right): \eta<m \leq M<\mu$ and $\eta<n \leq N<v$. Using the inequality $\sin x \leq x$ and $\sin y \leq \pi-y$, we get

$$
\left|\sum_{j=m}^{M} \sum_{k=n}^{N} c_{j k} \sin j x \sin k y\right| \leq x(\pi-y) \sum_{j=m}^{M} \sum_{k=n}^{N} j k\left|c_{j k}\right|<\frac{1}{\mu v} \sum_{j=m}^{\mu} \sum_{k=n}^{v} \epsilon \leq \epsilon .
$$

CASE $\left(b^{* *}\right): \max \{\eta, \mu\}<m \leq M$ and $\eta<n \leq N<v$. We obtain similarly as in case (b)

$$
\left|\sum_{j=m}^{M} \sum_{k=n}^{N} c_{j k} \sin j x \sin k y\right| \leq \sum_{k=n}^{N}|\sin k y| \cdot\left|\sum_{j=m}^{M} c_{j k} \sin j x\right| \leq(\pi-y) \sum_{k=n}^{N} k\left|\sum_{j=m}^{M} c_{j k} \sin j x\right|<(\pi \mathcal{C}+\pi) \epsilon .
$$

CASE $\left(c^{* *}\right): \eta<m \leq M<\mu$ and $\max \{\eta, v\}<n \leq N$. Applying (3.13) we get

$$
\begin{aligned}
\left|\sum_{j=m}^{M} \sum_{k=n}^{N} c_{j k} \sin j x \sin k y\right| & \leq \sum_{j=m}^{M}|\sin j x| \cdot\left|\sum_{k=n}^{N} c_{j k} \sin k y\right| \leq x \sum_{j=m}^{M} j\left|\sum_{k=n}^{N} c_{j k} \sin k y\right| \\
& \leq x \sum_{j=m}^{M} j \frac{\pi}{4(\pi-y)}\left(\sum_{k=n}^{N}\left|\Delta_{02} c_{j k}\right|+\sum_{k=N+1}^{N+2}\left|c_{j k}\right|+\sum_{k=n}^{n+1}\left|c_{j k}\right|\right)<(\pi \mathcal{C}+\pi) \epsilon .
\end{aligned}
$$

CASE $\left(d^{* *}\right): \max \{\eta, \mu\}<m \leq M$ and $\max \{\eta, v\}<n \leq N$. Using (3.12) and (3.13) and analogously as in case $(\mathrm{d})$, we get

$$
\begin{aligned}
& \mid \sum_{j=m}^{M} \sum_{k=n}^{N} c_{j k} \sin j x \sin k y \mid \leq \\
& \leq \frac{\pi^{2}}{16 x(\pi-y)}\left(\sum_{j=m}^{M} \sum_{k=n}^{N}\left|\Delta_{22} c_{j k}\right|+\sum_{j=M+1}^{M+2} \sum_{k=n}^{N}\left|\Delta_{02} c_{j k}\right|+\sum_{j=m}^{m+1} \sum_{k=n}^{N}\left|\Delta_{02} c_{j k}\right|+\sum_{j=m}^{M} \sum_{k=N+1}^{N+2}\left|\Delta_{20} c_{j k}\right|\right. \\
&\left.\quad+\sum_{j=M+1}^{M+2} \sum_{k=N+1}^{N+2}\left|c_{j k}\right|+\sum_{j=m}^{m+1} \sum_{k=N+1}^{N+2}\left|c_{j k}\right|+\sum_{j=m}^{M} \sum_{k=n}^{n+1}\left|\Delta_{20} c_{j k}\right|+\sum_{j=M+1}^{M+2} \sum_{k=n}^{n+1}\left|c_{j k}\right|+\sum_{j=m}^{m+1} \sum_{k=n}^{n+1}\left|c_{j k}\right|\right) \\
& \quad<\left(\frac{3}{2} \mathcal{C}+1\right) \pi^{2} \epsilon .
\end{aligned}
$$
Finally, let $x \in\left(\frac{\pi}{2}, \pi\right)$ and $y \in\left(\frac{\pi}{2}, \pi\right)$, set $\mu:=\left\lceil\frac{1}{\pi-x}\right\rceil$ and $v:=\left\lceil\frac{1}{\pi-y}\right\rceil$. Analogously as before we have four
cases: 
CASE $\left(a^{* * *}\right): \eta<m \leq M<\mu$ and $\eta<n \leq N<v$. Using the inequality $\sin x \leq \pi-x$ and $\sin y \leq \pi-y$, we get

$$
\left|\sum_{j=m}^{M} \sum_{k=n}^{N} c_{j k} \sin j x \sin k y\right| \leq(\pi-x)(\pi-y) \sum_{j=m}^{M} \sum_{k=n}^{N} j k\left|c_{j k}\right|<\frac{1}{\mu v} \sum_{j=m}^{\mu} \sum_{k=n}^{v} \epsilon \leq \epsilon .
$$

CASE $\left(b^{* * *}\right): \max \{\eta, \mu\}<m \leq M$ and $\eta<n \leq N<v$. We have similarly as in case $\left(b^{*}\right)$

$$
\left|\sum_{j=m}^{M} \sum_{k=n}^{N} c_{j k} \sin j x \sin k y\right| \leq(\pi-y) \sum_{k=n}^{N} k\left|\sum_{j=m}^{M} c_{j k} \sin j x\right|<(\pi \mathcal{C}+\pi) \epsilon .
$$

CASE $\left(c^{* * *}\right): \eta<m \leq M<\mu$ and $\max \{\eta, v\}<n \leq N$. We obtain similarly as in case $\left(c^{* *}\right)$

$$
\left|\sum_{j=m}^{M} \sum_{k=n}^{N} c_{j k} \sin j x \sin k y\right| \leq \leq(\pi-x) \sum_{j=m}^{M} j\left|\sum_{k=n}^{N} c_{j k} \sin k y\right|<(\pi \mathcal{C}+\pi) \epsilon .
$$

CASE $\left(d^{* * *}\right): \max \{\eta, \mu\}<m \leq M$ and $\max \{\eta, v\}<n \leq N$. Using (3.12) and (3.13), we have

$$
\begin{aligned}
& \left|\sum_{j=m}^{M} \sum_{k=n}^{N} c_{j k} \sin j x \sin k y\right| \leq \\
& \leq \frac{\pi^{2}}{16(\pi-x)(\pi-y)}\left(\sum_{j=m}^{M} \sum_{k=n}^{N}\left|\Delta_{22} c_{j k}\right|+\sum_{j=M+1}^{M+2} \sum_{k=n}^{N}\left|\Delta_{02} c_{j k}\right|+\sum_{j=m}^{m+1} \sum_{k=n}^{N}\left|\Delta_{02} c_{j k}\right|+\sum_{j=m}^{M} \sum_{k=N+1}^{N+2}\left|\Delta_{20} c_{j k}\right|\right. \\
& \left.\quad+\sum_{j=M+1}^{M+2} \sum_{k=N+1}^{N+2}\left|c_{j k}\right|+\sum_{j=m}^{m+1} \sum_{k=N+1}^{N+2}\left|c_{j k}\right|+\sum_{j=m}^{M} \sum_{k=n}^{n+1}\left|\Delta_{20} c_{j k}\right|+\sum_{j=M+1}^{M+2} \sum_{k=n}^{n+1}\left|c_{j k}\right|+\sum_{j=m}^{m+1} \sum_{k=n}^{n+1}\left|c_{j k}\right|\right) \\
& \quad<\left(\frac{3}{2} \mathcal{C}+1\right) \pi^{2} \epsilon
\end{aligned}
$$

If we summarize all partial estimations we get (5.2), this ends the proof of part (i).

Part (ii): Suppose that $\left\{c_{j k}\right\}_{j, k=1}^{\infty}$ is non-negative and let $\epsilon>0$ be arbitrarily fixed. Using the form (2.2) for the uniform regular convergence of (2.1), we find that there exists an integer $m_{0}=m_{0}(\epsilon)$ for which

$$
\left|\sum_{j=m}^{M} \sum_{k=n}^{N} c_{j k} \sin j x \sin k y\right|<\epsilon
$$

holds for any $m+n>m_{0}$ and any $(x, y)$. Set $x_{1}(m)=\frac{\pi}{4 m}, x_{2}(m)=\frac{\pi}{4 \lambda b_{1}(m)}, y_{1}(n)=\frac{\pi}{4 n}, y_{2}(n)=\frac{\pi}{4 \lambda b_{2}(n)}$ we have

$$
\begin{array}{lcccc}
\sin \left(j x_{1}(m)\right) \geq \sin \frac{\pi}{4} \quad \text { if } & m \leq j \leq 2 m+1 ; & \sin \left(j x_{2}(m)\right) \geq \sin \frac{\pi}{4 \lambda} & \text { if } & b_{1}(m) \leq j \leq 2 \lambda b_{1}(m) ; \\
\sin \left(k y_{1}(n)\right) \geq \sin \frac{\pi}{4} \quad \text { if } & n \leq k \leq 2 n+1 ; & \sin \left(k y_{2}(n)\right) \geq \sin \frac{\pi}{4 \lambda} \quad \text { if } & b_{2}(n) \leq k \leq 2 \lambda b_{2}(n) .
\end{array}
$$

Since $\left\{b_{1}(l)\right\}_{l=1}^{\infty},\left\{b_{2}(l)\right\}_{l=1}^{\infty},\left\{b_{3}(l)\right\}_{l=1}^{\infty}$ tends to infinity, there exists an $m_{1}$ such that for any $m, n: m+n>m_{1}$ implies $m+n>m_{0}, b_{1}(m)+n>m_{0}, m+b_{2}(n)>m_{0}$ and $b_{3}(m+n)>m_{0}$. Then by (5.3) and Lemma 3 , we have for $m+n>m_{1}$

$$
\begin{aligned}
(5 \mathcal{C}+8) \epsilon & >\mathcal{C}\left(\sup _{M+N \geq b_{3}(m+n)} \sum_{j=M}^{2 M} \sum_{k=N}^{2 N} c_{j k} \sin \left(j x_{1}(M)\right) \sin \left(k y_{1}(N)\right)\right) \\
& +2 \mathcal{C} \sum_{j=b_{1}(m)}^{2 \lambda b_{1}(m)} \sum_{k=n}^{2 n+1} c_{j k} \sin \left(j x_{2}(m)\right) \sin \left(k y_{1}(n)\right)+2 \mathcal{C} \sum_{j=m}^{2 m+1} \sum_{k=b_{2}(n)}^{2 \lambda b_{2}(n)} c_{j k} \sin \left(j x_{1}(m)\right) \sin \left(k y_{2}(n)\right) \\
& +8 \sum_{j=m}^{2 m+1} \sum_{k=n}^{2 n+1} c_{j k} \sin \left(j x_{1}(m)\right) \sin \left(k y_{1}(n)\right) .
\end{aligned}
$$


Next

$$
\begin{aligned}
(5 \mathcal{C}+8) \epsilon & >\mathcal{C}\left(\sin \frac{\pi}{4}\right)^{2}\left(\sup _{M+N \geq b_{3}(m+n)} \sum_{j=M}^{2 M} \sum_{k=N}^{2 N}\left|c_{j k}\right|\right)+2 \mathcal{C} \sin \frac{\pi}{4 \lambda} \sin \frac{\pi}{4} \sum_{j=b_{1}(m)}^{2 \lambda b_{1}(m)} \sum_{k=n}^{2 n+1} c_{j k} \\
& +2 \mathcal{C} \sin \frac{\pi}{4} \sin \frac{\pi}{4 \lambda} \sum_{j=m}^{2 m+1} \sum_{k=b_{2}(n)}^{2 \lambda b_{2}(n)} c_{j k}+8\left(\sin \frac{\pi}{4}\right)^{2} \sum_{j=m}^{2 m+1} \sum_{k=n}^{2 n+1} c_{j k}
\end{aligned}
$$

and finally, we have

$$
(5 \mathcal{C}+8) \epsilon>\left(\sin \frac{\pi}{4} \sin \frac{\pi}{4 \lambda}\right) m n c_{m n} \quad \text { whenever } \quad m+n>m_{1}: \text { and }: m, n>\lambda .
$$

Hence (2.2) is satisfied when $j+k \rightarrow \infty$ and $j, k \geq \lambda$. If $j \rightarrow \infty$ and $k<\lambda$ or $j<\lambda$ and $k \rightarrow \infty$, (2.2) follows from the uniform convergence of the series in (5.1). It completes the proof of part (ii).

\subsection{Proof of Theorem 8}

Part (i): We prove that $D G M\left({ }_{3} \alpha,_{3} \beta,,_{3} \gamma, 1\right) \subset D G M\left({ }_{3} \alpha,,_{3} \beta,,_{3} \gamma, 2\right)$. Let $\left\{c_{j k}\right\}_{j, k=1}^{\infty} \in D G M\left({ }_{3} \alpha,,_{3} \beta,,_{3} \gamma, 1\right)$. It easy to see that

$$
\begin{aligned}
\sum_{j=m}^{2 m-1}\left|\Delta_{20} c_{j n}\right| & \leq \sum_{j=m}^{\infty}\left|\Delta_{20} c_{j n}\right|=\sum_{j=m}^{\infty}\left|c_{j n}-c_{j+1, n}+c_{j+1, n}-c_{j+2, n}\right| \\
& \leq \sum_{j=m}^{\infty}\left|c_{j n}-c_{j+1, n}\right|+\sum_{j=m}^{\infty}\left|c_{j+1, n}-c_{j+2, n}\right| \leq 2 \sum_{j=m}^{\infty}\left|c_{j n}-c_{j+1, n}\right|=2 \sum_{j=m}^{\infty}\left|\Delta_{10} c_{j n}\right|
\end{aligned}
$$

and by (2.9), for $m \geq \lambda$ and $n \geq 1$,

$$
\begin{aligned}
\sum_{j=m}^{\infty}\left|\Delta_{10} c_{j n}\right| & =\sum_{r=0}^{\infty} \sum_{j=2^{r} m}^{2^{r+1} m-1}\left|\Delta_{10} c_{j n}\right| \leq \sum_{r=0}^{\infty} \frac{\mathcal{C}}{2^{r} m}\left(\sup _{M \geq b\left(2^{r} m\right)} \sum_{j=M}^{2 M}\left|c_{j n}\right|\right) \\
& \leq \sum_{r=0}^{\infty} \frac{1}{2^{r}}\left(\frac{\mathcal{C}}{m} \sup _{M \geq b(m)} \sum_{j=M}^{2 M}\left|c_{j n}\right|\right)=2 \cdot \frac{\mathcal{C}}{m} \sup _{M \geq b(m)} \sum_{j=M}^{2 M}\left|c_{j n}\right|
\end{aligned}
$$

Next, we get

$$
\sum_{j=m}^{2 m-1}\left|\Delta_{20} c_{j n}\right| \leq 4 \cdot \frac{\mathcal{C}}{m} \sup _{M \geq b(m)} \sum_{j=M}^{2 M}\left|c_{j n}\right| .
$$

Similarly as above, for $n \geq \lambda$ and $m \geq 1$,

$$
\sum_{k=n}^{2 n-1}\left|\Delta_{02} c_{m k}\right| \leq 4 \cdot \frac{\mathcal{C}}{n} \sup _{N \geq b(n)} \sum_{k=N}^{2 N}\left|c_{m k}\right| .
$$

Now, we have

$$
\begin{aligned}
\sum_{j=m}^{2 m-1} \sum_{k=n}^{2 n-1}\left|\Delta_{22} c_{j k}\right| & =\sum_{j=m}^{2 m-1} \sum_{k=n}^{2 n-1}\left|\Delta_{11} c_{j k}+\Delta_{11} c_{j+1, k}+\Delta_{11} c_{j, k+1}+\Delta_{11} c_{j+1, k+1}\right| \\
& \leq \sum_{j=m}^{\infty} \sum_{k=n}^{\infty}\left|\Delta_{11} c_{j k}\right|+\sum_{j=m+1}^{\infty} \sum_{k=n}^{\infty}\left|\Delta_{11} c_{j k}\right|+\sum_{j=m}^{\infty} \sum_{k=n+1}^{\infty}\left|\Delta_{11} c_{j k}\right|+\sum_{j=m+1}^{\infty} \sum_{k=n+1}^{\infty}\left|\Delta_{11} c_{j k}\right| \\
& \leq 4 \sum_{j=m}^{\infty} \sum_{k=n}^{\infty}\left|\Delta_{11} c_{j k}\right|
\end{aligned}
$$


and by (2.11), we obtain for $m, n \geq \lambda$

$$
\begin{aligned}
\sum_{j=m}^{\infty} \sum_{k=n}^{\infty}\left|\Delta_{11} c_{j k}\right| & =\sum_{r=0}^{\infty} \sum_{s=0}^{\infty} \sum_{j=2^{r} m}^{2^{r+1}} \sum_{j=2^{s} n}^{m-1}\left|\Delta_{11} c_{j k}\right| \leq \sum_{r=0}^{\infty} \sum_{s=0}^{\infty} \frac{\mathcal{C}}{2^{r} m \cdot 2^{s} n}\left(\sup _{M+N \geq b\left(2^{r} m+2^{s} n\right)} \sum_{j=M}^{2 M} \sum_{k=N}^{2 N}\left|c_{j k}\right|\right) \\
& \leq 4 \cdot \frac{\mathcal{C}}{m n}\left(\sup _{M+N \geq b(m+n)} \sum_{j=M}^{2 M} \sum_{k=N}^{2 N}\left|c_{j k}\right|\right) .
\end{aligned}
$$

Finally, we get

$$
\sum_{j=m}^{2 m-1} \sum_{k=n}^{2 n-1}\left|\Delta_{22} c_{j k}\right| \leq 16 \cdot \frac{\mathcal{C}}{m n}\left(\sup _{M+N \geq b(m+n)} \sum_{j=M}^{2 M} \sum_{k=N}^{2 N}\left|c_{j k}\right|\right) .
$$

From (5.4), (5.5) and (5.6) we have that $\left\{c_{j k}\right\}_{j, k=1}^{\infty} \in D G M\left({ }_{3} \alpha,,_{3} \beta,,_{3} \gamma, 2\right)$.

Part (ii): Let

$$
c_{j k}=\frac{2+(-1)^{j}}{j^{2}} \cdot \frac{2+(-1)^{k}}{k^{2}} \quad \text { for } j, k \in \mathbb{N} .
$$

We show that $\left\{c_{j k}\right\}_{j, k=1}^{\infty} \in D G M\left({ }_{3} \alpha,,_{3} \beta,,_{3} \gamma, 2\right)$. It easy to see that

$$
\Delta_{20} c_{j k}=c_{j k}-c_{j+2, k}=c_{j k} \cdot \frac{4(j+1)}{(j+2)^{2}}
$$

and

$$
\begin{aligned}
\sum_{j=m}^{2 m-1}\left|\Delta_{20} c_{j n}\right| & =\sum_{j=m}^{2 m-1}\left|c_{j n} \cdot \frac{4(j+1)}{(j+2)^{2}}\right| \leq \sum_{j=m}^{2 m-1}\left|c_{j n}\right| \frac{4}{j+1} \leq 4 \sum_{j=m}^{2 m-1}\left|c_{j n}\right| \frac{1}{j} \leq \frac{4}{m} \sum_{j=m}^{2 m-1}\left|c_{j n}\right| \leq \frac{4}{m} \sum_{j=m}^{2 m}\left|c_{j n}\right| \\
& \leq \frac{4}{m} \sup _{M \geq b(m)} \sum_{j=M}^{2 M}\left|c_{j n}\right| .
\end{aligned}
$$

Similarly as above

$$
\Delta_{02} c_{j k}=c_{j k}-c_{j, k+2}=c_{j k} \cdot \frac{4(k+1)}{(k+2)^{2}}
$$

and

$$
\sum_{k=n}^{2 n-1}\left|\Delta_{02} c_{m k}\right| \leq \frac{4}{n} \sup _{N \geq b(n)} \sum_{k=N}^{2 N}\left|c_{m k}\right|
$$

By elementary calculations

$$
\Delta_{22} c_{\mathrm{jk}}=c_{\mathrm{jk}}-c_{j+2, k-} c_{j, k+2}+c_{j+2, k+2}=c_{j k} \cdot \frac{4(j+1)}{(j+2)^{2}} \cdot \frac{4(k+1)}{(k+2)^{2}}
$$

and

$$
\begin{aligned}
\sum_{j=m}^{2 m-1} \sum_{k=n}^{2 n-1}\left|\Delta_{22} c_{j k}\right| & =\sum_{j=m}^{2 m-1} \sum_{k=n}^{2 n-1}\left|c_{j k} \cdot \frac{4(j+1)}{(j+2)^{2}} \cdot \frac{4(k+1)}{(k+2)^{2}}\right| \leq 16 \sum_{j=m}^{2 m-1} \sum_{k=n}^{2 n-1}\left|c_{j k}\right| \cdot \frac{1}{j+1} \cdot \frac{1}{k+1} \\
& \leq 16 \sum_{j=m}^{2 m-1} \sum_{k=n}^{2 n-1}\left|c_{j k}\right| \frac{1}{j k} \leq \frac{16}{m n} \sum_{j=m}^{2 m-1} \sum_{k=n}^{2 n-1}\left|c_{j k}\right| \leq \frac{16}{m n} \sum_{j=m}^{2 m} \sum_{k=n}^{2 n}\left|c_{j k}\right| \\
& \leq \frac{16}{m n} \sup _{M+N \geq b(m+n)} \sum_{j=M}^{2 M} \sum_{k=N}^{2 N}\left|c_{j k}\right| .
\end{aligned}
$$


Therefore $\left\{c_{j k}\right\}_{j, k=1}^{\infty} \in D G M\left({ }_{3} \alpha,_{3} \beta,_{3} \gamma, 2\right)$. Now, we show that $\left\{c_{j k}\right\}_{j, k=1}^{\infty} \notin D G M\left({ }_{3} \alpha,_{3} \beta,_{3} \gamma, 1\right)$. We have

$$
\begin{aligned}
\sum_{j=m}^{2 m-1}\left|\Delta_{10} c_{j n}\right| & =\sum_{j=m}^{2 m-1}\left|\frac{(-1)^{j}+2}{j^{2}} \cdot \frac{(-1)^{n}+2}{n^{2}}-\frac{(-1)^{j+1}+2}{(j+1)^{2}} \cdot \frac{(-1)^{n}+2}{n^{2}}\right| \\
& =\frac{(-1)^{n}+2}{n^{2}} \sum_{j=m}^{2 m-1}\left|\frac{(-1)^{j}+2}{j^{2}}-\frac{2-(-1)^{j}}{(j+1)^{2}}\right|
\end{aligned}
$$

Let $A_{m}=\{j: m \leq j \leq 2 m-1$ and $j$ is even $\}$. Then

$$
\sum_{j=m}^{2 m-1}\left|\Delta_{10} c_{j n}\right| \geq \frac{1}{n^{2}} \sum_{j \in A_{m}}\left(\frac{3}{j^{2}}-\frac{1}{(j+1)^{2}}\right) \geq \frac{1}{n^{2}} \sum_{j \in A_{m}}\left(\frac{3}{j^{2}}-\frac{1}{j^{2}}\right) \geq \frac{2}{n^{2}} \sum_{j \in A_{m}} \frac{1}{j^{2}} \geq \frac{m-1}{2 n^{2} m^{2}}
$$

and since

$$
\begin{aligned}
\frac{\mathcal{C}}{m} \sup _{M \geq b(m)} \sum_{j=M}^{2 M}\left|c_{j n}\right| & =\frac{\mathcal{C}}{m} \sup _{M \geq b(m)} \sum_{j=M}^{2 M}\left|\frac{(-1)^{j}+2}{j^{2}} \cdot \frac{(-1)^{n}+2}{n^{2}}\right| \leq \frac{\mathcal{C}}{m} \frac{(-1)^{n}+2}{n^{2}} \sup _{M \geq b(m)} \sum_{j=M}^{2 M}\left|\frac{(-1)^{j}+2}{j^{2}}\right| \\
& \leq \frac{3 \mathcal{C}}{m n^{2}} \sup _{M \geq b(m)} \sum_{j=M}^{2 M} \frac{3}{j^{2}} \leq \frac{9 \mathcal{C}}{m n^{2}} \sup _{M \geq b(m)} \frac{2}{M} \leq \frac{18 \mathcal{C}}{m n^{2} b(m)}
\end{aligned}
$$

the inequality

$$
\sum_{j=m}^{2 m-1}\left|\Delta_{10} c_{j n}\right| \leq \frac{\mathcal{C}}{m} \sup _{M \geq b(m)} \sum_{j=M}^{2 M}\left|c_{j n}\right|
$$

does not hold, because $\frac{1}{b(m)} \rightarrow 0$ as $m \rightarrow \infty$.

This ends the proof.

\subsection{Proof of Corollary 1}

Part (i): We prove that $\operatorname{DGM}\left({ }_{2} \alpha,,_{2} \beta,,_{2} \gamma, 1\right) \subset D G M\left({ }_{2} \alpha,{ }_{2} \beta, 2 \gamma, 2\right)$. Let $\left\{c_{j k}\right\}_{j, k=1}^{\infty} \in D G M\left({ }_{2} \alpha,,_{2} \beta, 2 \gamma, 1\right)$. Then for $m \geq \lambda$ and $n \geq 1$

$$
\begin{aligned}
& \sum_{j=m}^{2 m-1}\left|\Delta_{20} c_{j n}\right|=\sum_{j=m}^{2 m-1}\left|\Delta_{10} c_{j n}+\Delta_{10} c_{j+1, n}\right| \leq \sum_{j=m}^{2 m-1}\left|\Delta_{10} c_{j n}\right|+\sum_{j=m}^{2 m-1}\left|\Delta_{10} c_{j+1, n}\right| \\
& \leq \sum_{j=m}^{2 m-1}\left|\Delta_{10} c_{j n}\right|+\sum_{j=m+1}^{2 m}\left|\Delta_{10} c_{j n}\right| \leq 2 \sum_{j=m}^{2 m}\left|\Delta_{10} c_{j n}\right|+\left|\Delta_{10} c_{2 m, n}\right| \\
& \leq 2 \frac{\mathcal{C}}{m} \max _{b_{1}(m) \leq M \leq \lambda b_{1}(m)} \sum_{j=M}^{2 M} c_{j n}+\sum_{k=2 m}^{4 m-1}\left|\Delta_{10} c_{j n}\right| \\
& \leq 2 \frac{\mathcal{C}}{m}\left(\max _{b_{1}(m) \leq M \leq \lambda b_{1}(m)} \sum_{j=M}^{2 M} c_{j n}+\max _{b_{1}(2 m) \leq M \leq \lambda b_{1}(2 m)} \sum_{j=M}^{2 M} c_{j n}\right)=2 \frac{\mathcal{C}}{m}\left\{S_{1}+S_{2}\right\} .
\end{aligned}
$$

Let, for $m \geq \lambda, b_{1}^{\prime}(m)=b_{1}(m)$ if $S_{2} \leq S_{1}$ or $b_{1}^{\prime}(m)=b_{1}(2 m)$ if $S_{1} \leq S_{2}$. Then

$$
\sum_{j=m}^{2 m-1}\left|\Delta_{20} c_{j n}\right| \leq 4 \frac{\mathcal{C}}{m} \max _{b_{1}^{\prime}(m) \leq M \leq \lambda b_{1}^{\prime}(m)} \sum_{j=M}^{2 m} c_{j n}
$$

for $m \geq \lambda$ and $n \geq 1$. Similarly as above

$$
\sum_{k=n}^{2 n-1}\left|\Delta_{02} c_{m k}\right| \leq 4 \frac{\mathcal{C}}{n} \max _{b_{2}^{\prime}(n) \leq N \leq \lambda b_{2}^{\prime}(n)} \sum_{k=N}^{2 n} c_{m k}
$$


for $n \geq \lambda$ and $m \geq 1$. Finally, by (5.6) wet

$$
\sum_{j=m}^{2 m-1} \sum_{k=n}^{2 n-1}\left|\Delta_{22} c_{j k}\right| \leq 16 \cdot \frac{\mathcal{C}}{m n}\left(\sup _{M+N \geq b_{3}(m+n)} \sum_{j=M}^{2 M} \sum_{k=N}^{2 N}\left|c_{j k}\right|\right)
$$

for $m, n \geq \lambda$. From (5.8), (5.9) and (5.10) we get that $\left\{c_{j k}\right\}_{j, k=1}^{\infty} \in D G M\left({ }_{2} \alpha, 2 \beta,{ }_{2} \gamma, 2\right)$.

Part (ii): Taking the sequence (5.7), we can show, similarly as in the proof of Theorem 8 (part (ii)) that $\left\{c_{j k}\right\}_{j, k=1}^{\infty} \in D G M\left({ }_{2} \alpha, 2 \beta,,_{2} \gamma, 2\right)$ and $\left\{c_{j k}\right\}_{j, k=1}^{\infty} \notin D G M\left({ }_{2} \alpha, 2 \beta,,_{1} \gamma, 1\right)$.

It completes the proof.

\subsection{Proof of Remark 2}

Let $c_{j k}=a_{j} \cdot a_{k}$, where

$$
a_{n}=\left\{\begin{array}{lll}
\frac{3}{n \ln (n+1)} & \text { if } & n=3 l+1 \\
\frac{1}{n \ln (n+1)} & \text { if } & n \neq 3 l+1
\end{array}\right.
$$

for $l \in \mathbb{N} \cup\{0\}$ and $\left(x_{0}, y_{0}\right)=\left(\frac{2}{3} \pi, \frac{2}{3} \pi\right)$.

It is easy to see that (2.2) holds. Now, we shall prove that $\left\{c_{j k}\right\}_{j, k=1}^{\infty} \in D G M\left({ }_{3} \alpha,,_{3} \beta,_{3} \gamma, 3\right)$. Let

$$
A_{m}=\{j: j=3 l+1, l \in \mathbb{N} \cup\{0\}, m \leq j \leq 2 m-1\}
$$

and

$$
B_{m}=\{j: j \neq 3 l+1, l \in \mathbb{N} \cup\{0\}, m \leq j \leq 2 m-1\} .
$$

Then we have

$$
\begin{gathered}
\sum_{j=m}^{2 m-1}\left|\Delta_{30} c_{j n}\right|=\sum_{j=m}^{2 m-1} a_{n}\left|a_{j}-a_{j+3}\right| \\
=\sum_{j \in A_{m}} a_{n}\left(\frac{3}{j \ln (j+1)}-\frac{3}{(j+3) \ln (j+4)}\right)+\sum_{j \in B_{m}} a_{n}\left(\frac{1}{j \ln (j+1)}-\frac{1}{(j+3) \ln (j+4)}\right) \\
=3 \sum_{j \in A_{m}} a_{n}\left(\frac{j(\ln (j+4)-\ln (j+1))+3 \ln (j+4)}{j(j+3) \ln (j+1) \ln (j+4)}\right) \\
+\sum_{j \in B_{m}} a_{n}\left(\frac{j(\ln (j+4)-\ln (j+1))+3 \ln (j+4)}{j(j+3) \ln (j+1) \ln (j+4)}\right) .
\end{gathered}
$$

Applying the Lagrange theorem, for the function $y=\ln (x)$, there exists $c \in(j+4, j+1)$ such that

$$
\ln (j+4)-\ln (j+1)=\frac{3}{c} \leq \frac{3}{j+1}
$$

and

$$
\begin{aligned}
\sum_{j=m}^{2 m-1}\left|\Delta_{30} c_{j n}\right| & \leq 3 \sum_{j \in A_{m}} a_{n}\left(\frac{\frac{3 j}{j+1}+3 \ln (j+4)}{j(j+3) \ln (j+1) \ln (j+4)}\right)+\sum_{j \in B_{m}} a_{n}\left(\frac{\frac{3 j}{j+1}+3 \ln (j+4)}{j(j+3) \ln (j+1) \ln (j+4)}\right) \\
& \leq 3 \sum_{j \in A_{m}} a_{n}\left(\frac{3+3 \ln (j+4)}{j^{2} \ln (j+1) \ln (j+4)}\right)+\sum_{j \in B_{m}} a_{n}\left(\frac{3+3 \ln (j+4)}{j^{2} \ln (j+1) \ln (j+4)}\right) \\
& \leq 3 \sum_{j \in A_{m}} a_{n}\left(\frac{6}{j^{2} \ln (j+1)}\right)+\sum_{j \in B_{m}} a_{n}\left(\frac{6}{j^{2} \ln (j+1)}\right)=6 \sum_{j \in A_{m}} a_{n} a_{j} \frac{1}{j}+6 \sum_{j \in B_{m}} a_{n} a_{j} \frac{1}{j} \\
& \leq \frac{6}{m} \sum_{j=m}^{2 m}\left|c_{j n}\right| \leq \frac{6}{m} \sup _{M \geq b(m)} \sum_{j=M}^{2 M}\left|c_{j n}\right|
\end{aligned}
$$


Similarly as above

$$
\sum_{k=n}^{2 n-1}\left|\Delta_{03} c_{m k}\right| \leq \frac{6}{n} \sup _{N \geq b(n)} \sum_{k=N}^{2 n}\left|c_{m k}\right| .
$$

By elementary calculations we have

$$
\begin{aligned}
& \sum_{j=m}^{2 m-1} \sum_{k=n}^{2 n-1}\left|\Delta_{33} c_{j k}\right|=\sum_{j=m}^{2 m-1} \sum_{k=n}^{2 n-1}\left|\left(a_{j}-a_{j+3}\right)\left(a_{k}-a_{k+3}\right)\right|=\sum_{j=m}^{2 m-1}\left|a_{j}-a_{j+3}\right| \sum_{k=n}^{2 n-1}\left|a_{k}-a_{k+3}\right| \\
& =\left(\sum_{j \in A_{m}}\left(\frac{3}{j \ln (j+1)}-\frac{3}{(j+3) \ln (j+4)}\right)+\sum_{j \in B_{m}}\left(\frac{1}{j \ln (j+1)}-\frac{1}{(j+3) \ln (j+4)}\right)\right) \\
& \cdot\left(\sum_{k \in A_{n}}\left(\frac{3}{k \ln (k+1)}-\frac{3}{(k+3) \ln (k+4)}\right)+\sum_{k \in B_{n}}\left(\frac{1}{k \ln (k+1)}-\frac{1}{(k+3) \ln (k+4)}\right)\right) \\
& \leq\left(6 \sum_{j \in A_{m}} \frac{3}{j^{2} \ln (j+1)}+6 \sum_{j \in B_{m}} \frac{1}{j^{2} \ln (j+1)}\right)\left(6 \sum_{k \in A_{n}} \frac{3}{k^{2} \ln (k+1)}+6 \sum_{k \in B_{n}} \frac{1}{k^{2} \ln (k+1)}\right) \\
& =\left(6 \sum_{j \in A_{m}} c_{j} \frac{1}{j}+6 \sum_{j \in B_{m}} c_{j} \frac{1}{j}\right)\left(6 \sum_{k \in A_{n}} c_{k} \frac{1}{k}+6 \sum_{k \in B_{n}} c_{k} \frac{1}{k}\right) \leq \frac{36}{m n} \sum_{j=m}^{2 m-1} \sum_{k=n}^{2 n-1}\left|c_{j k}\right| \\
& \leq \frac{36}{m n} \sum_{M+N \geq b(m+n)}^{2 M} \sum_{j=M}^{2 N}\left|c_{j k}\right| .
\end{aligned}
$$

From (5.11), (5.12) and (5.13) we obtain that $\left\{c_{j k}\right\}_{j, k=1}^{\infty} \in \operatorname{DGM}\left({ }_{3} \alpha,{ }_{3} \beta,{ }_{3} \gamma, 3\right)$.

Moreover, we have

$$
\begin{aligned}
& \sum_{j=1}^{3 M+2} \sum_{k=1}^{3 N+2} c_{j k} \sin j x_{0} \sin k y_{0} \\
= & \left(a_{1} \sin \frac{2}{3} \pi+a_{2} \sin \frac{4}{3} \pi+\sum_{j=3}^{3 M+2} a_{j} \sin j x_{0}\right)\left(a_{1} \sin \frac{2}{3} \pi+a_{2} \sin \frac{4}{3} \pi+\sum_{k=3}^{3 N+2} a_{k} \sin k y_{0}\right) \\
= & {\left[\left(a_{1} \sin \frac{2}{3} \pi-a_{2} \sin \frac{2}{3} \pi\right)+\sum_{j=1}^{M} \sum_{i=0}^{2} a_{3 j+i} \sin \left((3 j+i) \frac{2}{3} \pi\right)\right] } \\
\cdot & \left.\left(a_{1} \sin \frac{2}{3} \pi-a_{2} \sin \frac{2}{3} \pi\right)+\sum_{k=1}^{N} \sum_{i=0}^{2} a_{3 k+i} \sin \left((3 k+i) \frac{2}{3} \pi\right)\right] \\
= & \left.\sin \frac{2}{3} \pi\left(a_{1}-a_{2}\right)+\sum_{j=1}^{M}\left(a_{3 j+1} \sin \frac{2}{3} \pi+a_{3 j+2} \sin \frac{4}{3} \pi\right)\right] \cdot\left[\sin \frac{2}{3} \pi\left(a_{1}-a_{2}\right)+\sum_{k=1}^{N}\left(a_{3 k+1} \sin \frac{2}{3} \pi+a_{3 k+2} \sin \frac{4}{3} \pi\right)\right] \\
= & \left(\sin \frac{2}{3} \pi\right)^{2} \sum_{j=0}^{M} \sum_{k=0}^{N}\left(\frac{3}{(3 j+1) \ln (3 j+2)}-\frac{1}{(3 j+2) \ln (3 j+3)}\right) \cdot\left(\frac{2}{(3 k+1) \ln (3 k+2)}-\frac{1}{(3 k+2) \ln (3 k+3)}\right) \\
\geq & \left(\sin \frac{2}{3} \pi\right)^{2} \sum_{j=0}^{M} \sum_{k=0}^{N} \frac{2}{(3 j+1) \ln (3 j+3)} \cdot \frac{2}{(3 k+1) \ln (3 k+3)} \rightarrow \infty \quad \operatorname{as} \quad M+N \rightarrow \infty
\end{aligned}
$$

This ends the proof. 


\section{References}

[1] T. W. Chaundy and A. E. Jolliffe, The uniform convergence of certain class of trigonometrical series, Proc. London Math. Soc., 15 (1916), 214-216.

[2] P. KóRus, Remarks on the uniform and $L^{1}$-convergence of trigonometric series, Acta Math. Hungar., 128 (2010), 369-380.

[3] P. Kórus and F. Móricz, On the uniform convergence of double sine series, Studia Math., 193 (2009), 79-97.

[4] P. KóRus, On the uniform convergence of double sine series with generalized monotone coefficients, Periodica Math. Hungar., 63 (2011), 205-214.

[5] L. LeIndLeR, On the uniform convergence of single and double sine series, Acta Math. Hungar., 140 (2013), $232-242$.

[6] B. SzAL, A new class of numerical sequences and its applications to uniform convergence of sine series, Math. Nachr., 284, No 14-15(2011), 1985-2002.

[7] B. Szal, On L-convergence of trigonometric series, J. Math. Anal. Appl., 373 (2011), 449-463.

[8] S. Tiknonov, Trigonometric series with general monotone coefficients, J. Math. Anal. Appl., 326(1) (2011), 721-735.

[9] D. S. YU and S. P. Zhou, A generalization of monotonicity condition and applications, Acta Math. Hungar., 115 (2007), 247-267.

[10] S. P. Zhоu, P. Zhou and D. S. Yu, Ultimate generalization to monotonicity for uniform convergence of trigonometric series, Sci. China Math., 53(7) (2010), 1853-1862.

[11] I. E. ŽAK and A. A. ŠNEIDER, Conditions for uniform convergence of double sine series, Izv. Vyš̌. Učebn. Zaved. Matematika, 4 (1966), 44-52, in Russian. 